\title{
Compartment models for estimating attributes of digesta flow in cattle*
}

\author{
BY K. R. POND† AND W. C. ELLIS§ \\ Department of Animal Science, Texas $A \& M$ University, College Station, \\ Texas 77843, USA
}

AND J. H. MATIS

Department of Statistics, Texas A \& M University, College Station, Texas 77843, USA

AND H. M. FERREIRO $\ddagger$ AND J. D. SUTTON

AFRC Institute for Grassland and Animal Production, Hurley, Maidenhead SL6 5LR

(Received 8 February 1988-Accepted 20 June 1988)

1. The basic assumptions involved in one- and two-compartment models with age-independent distributed residence times (exponential, Gl) for describing digesta flow are reviewed as the bases for describing families of one- and two-compartment models which assume age-dependent distributions $(\mathrm{G} n)$ of residence times.

2. The two-compartment, age-independent model with exponentially distributed residence times (G1G1) yielded estimates of essentially equal rate parameters when fitted to faecal values for all four cows receiving a diet of $500 \mathrm{~g}$ coarsely chopped, sodium hydroxide-treated straw $/ \mathrm{kg}$ and one of four cows receiving the same diet but with ground and pelleted straw. The incorporation of progressively higher orders of age dependency $(\mathrm{G} 2-\mathrm{G} 6, \mathrm{G} n)$ into the faster turnover compartment of two-compartment models $(\mathrm{G} n \mathrm{Gl})$ resulted in a resolution of equal rate parameters estimated by the G1Gl model and a reduction in standard errors for the rate and the initial concentration parameters.

3. The occurrence of equal rate parameters in two-compartment models indicated an age-dependent process; a process which could equally well be described by a one-compartment, age-dependent compartment having an order of age dependency equal to the sum of these orders in the two-compartment model with equal rate parameters.

4. The age-independent models overestimated time of first appearance in the faeces of a meal's particles. The association of age dependency with the faster turnover compartment resulted in earlier estimates for first appearance of the marked particles; estimates which were more consistent with observed first appearance.

5 . The faecal excretion pattern from cows fed on the ground and pelleted straw diet exhibited an ageindependent distribution of longer residence times which dominated approximately $80 \%$ of the later residence times. Age-dependent, one-compartment models gave a poor fit to such data from these cows fed on ground and pelleted straw. In contrast, age-dependent, one-compartment models provided an excellent fit to data from cows fed on chopped straw; data which indicated that age-independent distributions of residence times were much delayed in appearing or were totally absent.

6. The mean residence time for the slower turnover, age-independent compartment estimated from faecal excretional of stained particles from either diet was similar to that estimated from duodenal concentrations of the stained particles. This suggests that the slower turnover model compartment was confined to preduodenal sites.

7. The mean residence time for the faster turnover, age-dependent compartment estimated from duodenal data was $58-62 \%$ that estimated from faecal data and suggests that the site of this model compartment was both preand post-duodenal.

8. It is emphasized that the slow and imperfect mixing of particulate matter that occurs in reticulo-rumen digesta is inconsistent with the assumptions of instantaneous and homogeneous mixing made by models having age-independent distributions of residence times. The use of age-dependent distributed residence times can accommodate such imperfect mixing and is consistent with the existence of age-discriminating processes involved in particle flow from the reticulo-rumen. Age dependency also offers improved precision in estimating parameters of digesta flow via processes having inherent uncertainty in their mixing and age-discriminating mechanisms.

* Approved for publication as TA 22814 by the Director of the Texas Agricultural Experiment Station.

Present addresses : $\uparrow$ Department of Animal Science, North Carolina State University, Box 7621, Raleigh North Carolina 27695-7621, USA and ¥Institute de Investigaciones Pecuarias SARH, Direccion Regional - Zona Norte, Apartado Postal no. 682, Chihuahua - Chih, Mexico.

$\S$ For reprints. 
(a) One-compartment, unidirectional flow

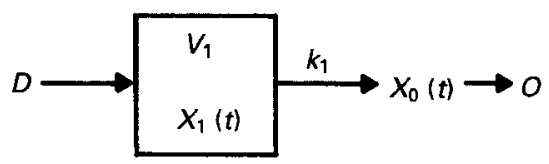

(b) Two-serial-compartment, unidirectional flow

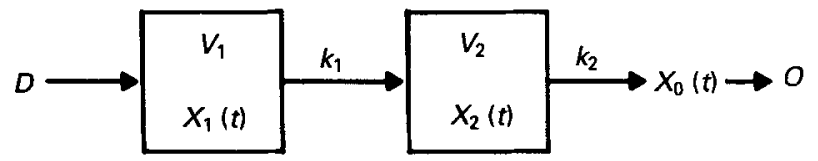

Fig. 1. Diagrammatic representation of $(a)$ one- and $(b)$ two-compartment models. (a) $D$, pulse dose of marker; $V_{1}$, digesta volume of compartment $1 ; X_{1}(t)$, dose remaining in compartment 1 at time $t$; $X_{0}(t)$, dose that has passed out of $V_{1}$ by time $t ; k_{1}$, age-independent turnover-rate parameter for compartment 1; $O$, output of $D$ from compartmental system. $(b) D, V_{1}, X_{1}(t)$ and $k_{1}$ as in $(a) ; V_{2}$, digesta volume of compartment $2 ; X_{2}(t)$, dose remaining in compartment 2 at time $t ; k_{2}$, age-independent turnover-rate parameter for compartment $2 ; O$, output of $D$ from compartmental system.

A number of investigators have observed and described the passage of digesta through the gastrointestinal tract of ruminants (Balch, 1950; Blaxter et al. 1956; Castle, 1956; Brandt \& Thacker 1958; Grovum \& Williams, 1973; Ellis et al. 1979; Faichney \& Boston, 1983; Faichney, 1984; Dhanoa et al. 1985; France et al. 1985), rats (Marcus \& Lengemann, 1962) and human beings (Luckey et al. 1977, 1979). In general; the biological and mathematical assumptions involved have not been emphasized and the models have estimated only passage parameters (Warner, 1981). Stochastic models have been developed for biological systems (Matis \& Wehrly, 1979; Matis \& Tolley, 1980; Matis, 1987) and appear applicable to digesta passage data. The purpose of the present paper is to utilize a compartmental analysis approach to develop and interrelate systematically several models and illustrate some of their applications and limitations in expressing and interpreting digesta flow. The uses of these models and their interrelations are illustrated with data from the gastrointestinal tract of the ruminant.

\section{EXPERIMENTAL METHODS}

\section{Development of models}

General concepts, terminology and assumptions. Fig. 1 diagrammatically portrays the flow of digesta through one- and two-compartment systems. In the one-compartment system, the dose of an indigestible component of feed particles, $D$, enters compartment 1 with volume $V_{1}$, and mixes with existing digesta particles of various ages in and derived from a succession of previous meals. This mixing of newly ingested particles and particles of various ages results in competition for exit flow from $V_{1}$ between the aged particles and newly ingested particles which are otherwise capable of exiting the compartment. If equal probability exists for exit flow from $V_{1}$ of particles of all ages, including newly ingested particles, then the $D$ dosed particles have exponentially distributed residence times and the proportion of $D$ remaining in compartment 1 at time $t$, denoted $X_{1}(t)$, which exits per unit time is a constant, $k_{1}$, throughout the excretion period. In the present paper, models assuming equal probability for escape of all particles will be referred to as age-independent models since their fractional turnover rate, $k_{1}$, is independent of the age or, alternatively, past residence time of the particle in the compartment. 

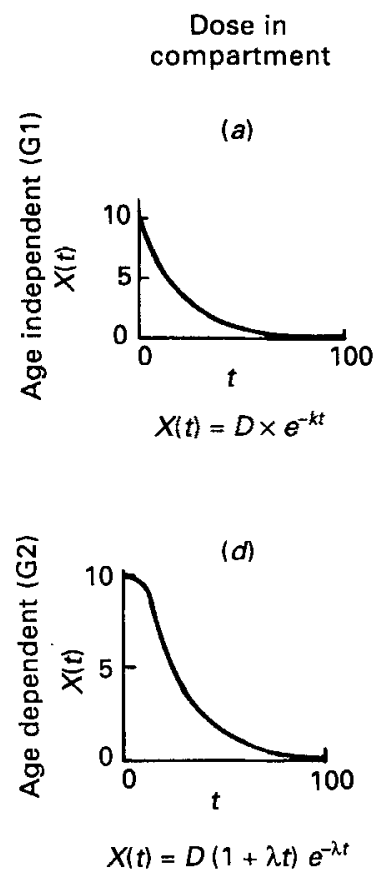

\section{Concentration emerging from compartment}

(b)

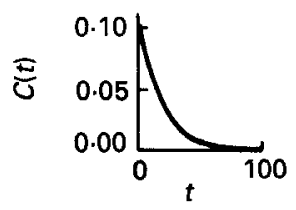

$C(t)=\frac{D}{V} \times e^{-k t}$

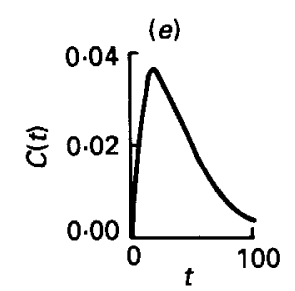

$C(t)=\frac{D}{V} \times \lambda \times t \times e^{-\lambda t}$
Proportion emerging from compartment

(c)

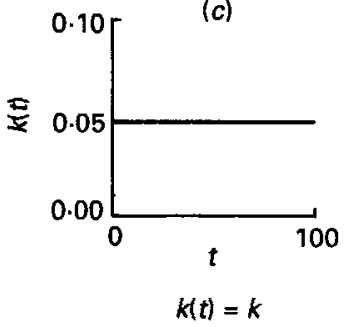

(f)

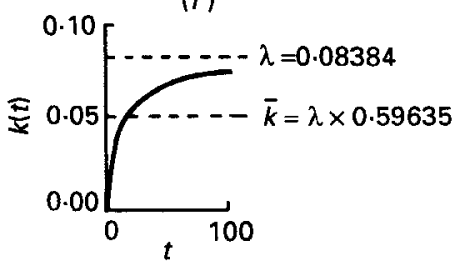

$k(t)=\lambda^{2} \times t /(1+\lambda t)$

Fig. 2. Graphic comparison of various expressions for age-independent $(a, b$ and $c$ ) and age-dependent $(d, e$ and $f)$ models for dose $(D)$ remaining in dosed compartment $(a$ and $d$ ), concentration $(C)$ emerging from dosed compartment ( $b$ and $e$ ) and proportion $(k(t))$ emerging from dosed compartment $(c$ and $f)$ of digesta volume $V$. The curves were generated for fixed parameters of $D=10, V=100, k=0.05$ and the age-dependent rate parameter $(\lambda)$ of 0.08348 .

The two-compartment models include a second compartment, having a digesta volume of $V_{2}$, which is the recipient of the diluting, turnover flow from $V_{1}$. The $D$ exiting compartment 1 then enters compartment 2 and mixes with digesta particles in compartment 2 which have been derived from a succession of preceding flows from compartment 1. Assumptions of equal probability for exit flow for all particles from compartment 2 (or an exponential distribution of residence times) leads to an age-independent rate-constant, $k_{2}$, for the diluting, turnover flow from compartment 2 in a similar manner as described for compartment 1 .

Fig. 2(a,b,c) compares the dose remaining in the compartment, the concentration emerging from the compartment and the proportion emerging from a single, ageindependent compartment ( $\mathrm{Gl}$, an exponential distribution of residence times).

A number of processes involved in particle flow from the reticulo-rumen (Sutherland, 1988) suggest that such flow may not be a simple first-order, age-independent process. Rather, an age-dependent process has been suggested in which the probability for escape of a particle increases with its residence time or, alternatively, age in the reticulo-rumen. Matis (1972) has suggested the use of a gamma distribution with integer-shape parameters (Erlang) of residence times to model such an age-dependent process. Although other distributions (e.g. log normal or Weibull) may also be appropriate to represent this agedependent process, the family of gamma distributions offers wide flexibility in form for this application. For the assumed gamma distribution of residence times, the rate function is initially zero and increases with time until it reaches a constant rate asymptote (Fig. 2(f)). 


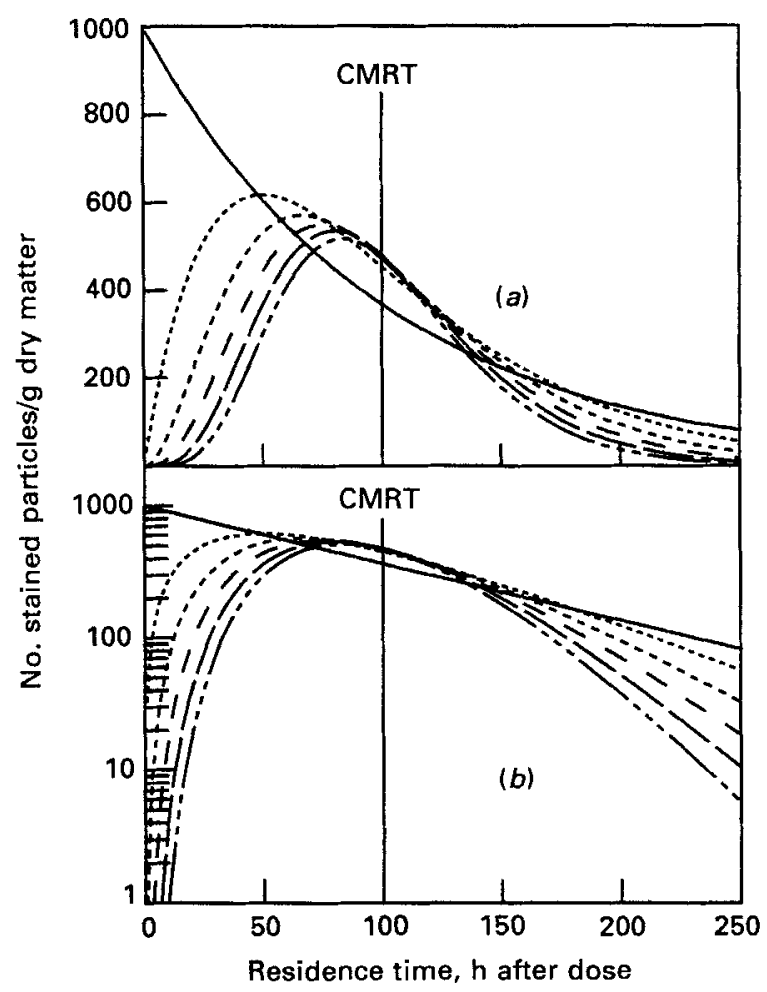

Fig. 3. The effect of increasing order of gamma distribution (G1 to G6) in one-compartment models on excretion patterns of particles having a compartmental mean retention time (CMRT) of $100 \mathrm{~h}$ plotted arithmetically $(a)$ and semi-logarithmically $(b) .(-), \mathrm{G} 1$ or exponential; $(-\ldots), \mathrm{G} 2 ;(---), \mathrm{G} 3$; $(-\ldots), \mathrm{G} 4 ;\left({ }_{-}\right), \mathrm{GS} ;(-\ldots \ldots)$, G6. Note the effect of increasing order of gamma distribution $(\mathrm{G} 2-\mathrm{G} 6)$ on progressively retarding the rate of emergence of particles from the compartment.

Age dependency can be included in one- or two-compartment models. To avoid confusion, age-dependent rate parameters are signified by lambda, $\lambda$, and age-independent rate parameters are signified by $k$. Note that the age function, $k(t)$, is a constant for the ageindependent (G1) compartment (Fig. 2(c)). In contrast, note that for a gamma $2(\mathrm{G} 2)$ distribution of residence times, the $k(t)$ rate function increases with residence time of the particle $(t)$ to an asymptotic rate parameter, $\lambda$, which is the age-dependent (G2) rate parameter.

In the age-independent (G1) model, the departure rate is $k$ for all particles regardless of their previous residence times. Therefore the mean rate at which particles are leaving is $k$, and the mean residence time is $1 / k$. In the age-dependent $(\mathrm{G} 2)$ model, the mean residence time is $2 / \lambda$. The rate is a function of the past residence time, $t$, and it is shown subsequently that the mean departure rate averaged over particles of all past residence times is a constant function of $\lambda$ (i.e., $0.59635 \lambda$ for G2 (Fig. $2(f)$ ).

Increasing orders of age dependency can be inserted into the model by using increasing orders of integer gamma distributions $(\mathrm{G} 2, \mathrm{G} 3, \ldots, \mathrm{G} n)$. The consequences of using increasing orders of gamma distribution $(n)$ to fit the concentration of marked, dosed particles emerging from a one-compartment model having a mean residence time of 100 $h$ is shown in Fig. 3. With instantaneous mixing of the dose $D$ and an exponential distribution of residence times (G1), emergence of marked particles is immediate and results in an initial maximum concentration of marked particles which then declines 
Table 1. Total dose remaining in one-compartment models with various gamma residencetime distributions

\begin{tabular}{cll}
\hline Model & $\begin{array}{c}\text { Residence-time } \\
\text { distribution* }\end{array}$ & $\begin{array}{c}\text { Total dose }(D) \text { remaining } \\
\text { in system } \dagger\end{array}$ \\
\hline G1 & Exponential $\ddagger$ & $D e^{-k t}$ \\
G2 & Gamma-2 & $D e^{-\lambda t}(1+\lambda t)$ \\
G3 & Gamma-3 & $D e^{-\lambda t}\left[1+\lambda t+(\lambda t)^{2} / 2\right]$ \\
Gn & Gamma- $n$ & $D e^{-\lambda t} \sum_{i=0}^{n-1}(\lambda t)^{i} / i !$ \\
\hline
\end{tabular}

$k$, Rate parameter for exponentially distributed residence times; $t$, time after dose of marker; $\lambda$, rate parameter for gamma-distributed residence times.

* Parameter $n, n=1,2, \ldots$, is called order of age dependency.

$\dagger$ To incorporate time delay $(\tau)$, substitute $(t-\tau)$ for $t$.

$\ddagger$ Exponential is equivalent to gamma-1.

Table 2. Fractional concentration of marker in material emerging from one-compartment models with various gamma residence-time distributions

\begin{tabular}{ccll}
\hline Model & $\begin{array}{c}\text { Residence-time } \\
\text { distribution* }\end{array}$ & Marker concentration $\dagger$ \\
\hline G1 & Exponential & $C e^{-k t}$ \\
G2 & Gamma-2 & $C \lambda t e^{-\lambda t} / 0.59635$ \\
G3 & Gamma-3 & $C \lambda^{2} t^{2} e^{-\lambda t} /(2 \times 0.47454)$ \\
G4 & Gamma-4 & $C \lambda^{3} t^{3} e^{-\lambda t} /(6 \times 0.40857)$ \\
G5 & Gamma-5 & $C \lambda^{4} t^{4} e^{-\lambda t} /(24 \times 0.36528)$ \\
G6 & Gamma-6 & $C \lambda^{5} t^{5} e^{-\lambda t} /(120 \times 0.33929)$ \\
\hline
\end{tabular}

$C$, Initial fractional concentration in compartment volume $(V)$ assuming instantaneous mixing of dose $(D)$, i.e. $C=D / V ; k$, rate parameter for exponentially distributed residence times, $t$, time after dose of marker; $\lambda$, rate parameter for gamma-distributed residence times.

* Parameter $n, n=1,2, \ldots$, is called order of age dependency.

$\dagger$ To incorporate time delay $(\tau)$, substitute $(t-\tau)$ for $t$.

exponentially as illustrated by the linear nature of this distribution when plotted on a semilogarithmic scale in Fig. 3(b). In contrast to age-independent flow (G1), progressively increasing the order of the gamma distribution results in a progressive delay in outflow during early residence times and progressive increases in proportional outflow during later residence times as $k(t)$ approaches the asymptotic rate. These characteristics of varying order of gamma distribution are most clearly contrasted by the semi-logarithmic plot of Fig. $3(b)$. Increasing order of gamma distribution results in a progressive departure from an instantaneously mixed, age-independent-distributed residence time compartment (linear descending segment in the semi-logarithmic plot).

One-compartment models. One-compartment models with various residence-time distributions for the total dose remaining in the compartmental system are summarized in Table 1. Models with various residence-time distributions for the concentration of marked particles emerging from a single compartment are summarized in Table 2 . In the models expressing marker emergence (Table 2), different constants are included in the agedependent models (G2-G6) so that the value estimated for the initial concentration of marker within the single compartment, $C$, will be equivalent for all age-dependent and age- 
independent models. This is necessary since the initial concentration in the age-dependent compartment cannot be estimated as the initial concentration emerging from the compartment since this is zero (Fig. 2(e)). Rather, $C$ can be estimated from the mean flowrate $(\bar{k}$, Fig. $2(f))$ which represents the mean rate at which all particles emerged from the age-dependent compartment during the entire period of measurement. The mean flow-rate from an age-dependent compartment is the integral of the product of the rate function and the distribution of particles of different resident ages emerging from the system. The rate function for $\mathrm{G} 2$ (Fig. $2(f)$ ) is:

$$
k(t)=\lambda^{2} t /(1+\lambda t)
$$

The distribution of particles emerging from the $\mathrm{G} 2$, age-dependent compartment (derivative of concentration in Fig. $2(e))$ is:

$$
f(t)=\lambda^{2} t e^{-\lambda t}
$$

Integration of eqns (1) and (2) yields:

$$
\int k(t) f(t) d t=\int_{0}^{\infty} \lambda^{4} t^{2} e^{-\lambda t} /(1+\lambda t) d t=0.59635 \lambda .
$$

Note that for an age-independent compartment similar calculations show that the mean rate is, as expected, equal to $k$ :

$$
\int k^{2} e^{-k t}=k
$$

The same approach was used to compute constants $(F)$ to estimate the mean flow-rate from $\lambda$ estimated by one-compartment models with G3, G4, G5 and G6 age dependency and yield $0.47454,0.40857,0.365276$ and 0.339291 respectively for values of $F$. Incorporation of these constants $(F)$ into the one-compartment, age-dependent models of marker concentration in the output (Table 2) results in equivalent meaning for $C$ and allows the direct computation of $V$ as equalling $D / C$ from any model. Total output rate from the G1 model equals $V \times k$. However, output rate computed from the age-dependent models requires the mean flow-rate $(\bar{k}=F \times \lambda)$. For example, output computed from the G2 model equals $V \times 0.59635 \lambda$ and for the $\mathrm{G} n$ model equals $V \times F \times \lambda$.

The model has a somewhat simpler form if the objective is only the computation of output (G. R. Reese, personal communication). In this case, the output, $O$, may be estimated directly from the following alternative form of the equations in Table 2 for the marker concentration of $\mathrm{G} n$ at time $t$ :

$$
(D / O) \lambda^{n} t^{n-1} e^{-\lambda t} /(n-1) \text { ! }
$$

Two-compartment models. Matis (1972) included gamma age dependency into one of the compartments in the two-compartment, age-independent model proposed by Blaxter et al. (1956). In the Matis (1972) model, $\lambda_{1}$ is an age-dependent rate parameter for flow emerging from the age-dependent compartment and $k_{2}$ is the age-independent flow-rate from the ageindependent compartment. The two-compartment model with gamma-2 age dependency and gamma-1 age independence is abbreviated G2G1. Table 3 presents two-compartment models with various orders of age dependency for total dose in the two-compartment system (see Matis (1972) for details on the derivation of these expressions).

Table 4 presents two-compartment models with various orders of age dependency for concentration of marker emerging from the two-compartment system. It should be noted that two-compartment models do not specify the order or relative size of the two sequential compartments. The use of differences in structure for age-dependent and age-independent sequential compartment provides the statistical opportunity to characterize age dependency 
Table 3. Total dose remaining in two-compartment models (compartments 1 and 2) with various gamma residence-time distributions in compartment 1

\begin{tabular}{|c|c|c|c|}
\hline \multirow[b]{2}{*}{ Model } & \multicolumn{2}{|c|}{$\begin{array}{l}\text { Residence-time } \\
\text { distribution }\end{array}$} & \multirow[b]{2}{*}{ Total dose remaining in system* } \\
\hline & Compartment 1 & Compartment 2 & \\
\hline $\begin{array}{l}\text { G1G1 } \\
\text { G2G1 } \\
\text { G3G1 }\end{array}$ & $\begin{array}{l}\text { Exp. } \dagger \\
\text { Gamma-2 } \\
\text { Gamma-3 }\end{array}$ & $\begin{array}{l}\text { Exp. } \\
\text { Exp. } \\
\text { Exp. }\end{array}$ & $\begin{array}{l}D\left(k_{1} e^{-k_{2} t}-k_{2} e^{-k_{1} t}\right) /\left(k_{1}-k_{2}\right) \\
D\left\{\delta^{2} e^{-k_{2} t}+e^{-\lambda_{1} t}\left[1-\delta^{2}+(1-\delta) \lambda_{1} t\right]\right\} \\
D\left\{\delta^{3} e^{-k_{2} t}+e^{-\lambda_{1} t}\left[1-\delta^{3}+\left(1-\delta^{2}\right) \lambda_{1} t+(1-\delta)\left(\lambda_{1} t\right)^{2} / 2\right]\right\}\end{array}$ \\
\hline $\mathrm{G} n \mathrm{G} 1$ & Gamma- $n$ & Exp. & $D\left[\delta^{n} e^{-k_{2} t}+e^{-\lambda_{1} t} \sum_{i=0}^{n-1}\left(1-\delta^{n-i}\right)\left(\lambda_{1} t\right)^{i} / i !\right]$ \\
\hline
\end{tabular}

$D$, Initial dose; Exp., exponential $k_{2}$, rate parameter for exponentially distributed residence times; 1 , time after dose of marker; $\lambda_{1}$, rate parameter for gamma-distributed residence times; $\delta=\lambda_{1} /\left(\lambda_{1}-k_{2}\right)$.

* To incorporate time delay $(\tau)$, substitute $(t-\tau)$ for $t$.

+ Exponential is equivalent to gamma- 1 .

Table 4. Fractional concentration of marker in material emerging from two-compartment (compartments $I$ and 2) models with various gamma residence-time distributions in compartment 1

\begin{tabular}{|c|c|c|c|}
\hline \multirow[b]{2}{*}{ Model } & \multicolumn{2}{|c|}{$\begin{array}{l}\text { Residence-time } \\
\text { distribution }\end{array}$} & \multirow[b]{2}{*}{ Marker concentration* } \\
\hline & Compartment 1 & Compartment 2 & \\
\hline $\begin{array}{l}\text { G1G1 } \\
\text { G2G1 } \\
\text { G3G1 }\end{array}$ & $\begin{array}{l}\text { Exp. } \dagger \\
\text { Gamma-2 } \\
\text { Gamma-3 }\end{array}$ & $\begin{array}{l}\text { Exp. } \\
\text { Exp. } \\
\text { Exp. }\end{array}$ & $\begin{array}{l}C_{2} k_{1}\left(e^{-k_{2} t}-e^{-k_{1} t}\right) /\left(k_{1}-k_{2}\right) \\
C_{2}\left[\delta^{2} e^{-k_{2} t}-e^{-\lambda_{1} t}\left(\delta^{2}+\delta \lambda_{1} t\right)\right] \\
C_{2}\left[\delta^{3} e^{-k_{2} t}-e^{-\lambda_{1} t}\left(\delta^{3}+\delta^{2} \lambda_{1} t+\delta \lambda_{1}^{2} t^{2} / 2\right)\right]\end{array}$ \\
\hline $\mathrm{G} n \mathrm{Gl}$ & Gamma- $n$ & Exp. & $C_{2}\left[\delta^{n} e^{-k_{2} t}-e^{-\lambda_{2} t} \sum_{i=1}^{n} \delta^{i}\left(\lambda_{1} t\right)^{n-i} /(n-i) !\right.$ \\
\hline
\end{tabular}

$C_{2}$, initial concentration in second compartment if dose $D$ had been introduced into volume $V$ at second compartment and instantaneously mixed, i.e. $C_{2}=D / V ; k_{1}$, rate parameter for exponentially distributed residence times; $t$, time after dose of marker; $\lambda_{1}$, rate parameter for gamma-distributed residence times; Exp., exponential; $\delta=\lambda_{1} /\left(\lambda_{1}-k_{2}\right)$.

* To incorporate time delay $(\tau)$, substitute $(t-\tau)$ for $t$.

$\dagger$ Exponential is equivalent to gamma-1.

with either the faster (smaller) or slower (larger) compartment. Experience has indicated that age dependency is consistently associated with the faster turnover compartment (Matis, 1972; Ellis et al. 1984).

If it is assumed that the faster turnover, age-dependent compartment is the first compartment encountered by feed residues, then marker exits the system from the ageindependent compartment (G1) of the two-compartment system. Therefore, $C_{2}$ represents the initial concentration of the marker in this terminal compartment with exponentially distributed residence time so that the volume of undigested material in the compartment then equals $D / C_{2}$. Since the output of compartment 1 also sequentially passes through compartment 2 , the output from the system of compartment 1 or compartment 2 each equals $\left(D / C_{2}\right) \times k_{2}$. Since the outputs of indigestible $D$ from each of the two sequential compartments are equal to each other and to that of the total system, then the volume of compartment 1 is related to the difference in the mean turnover for the two compartments. 


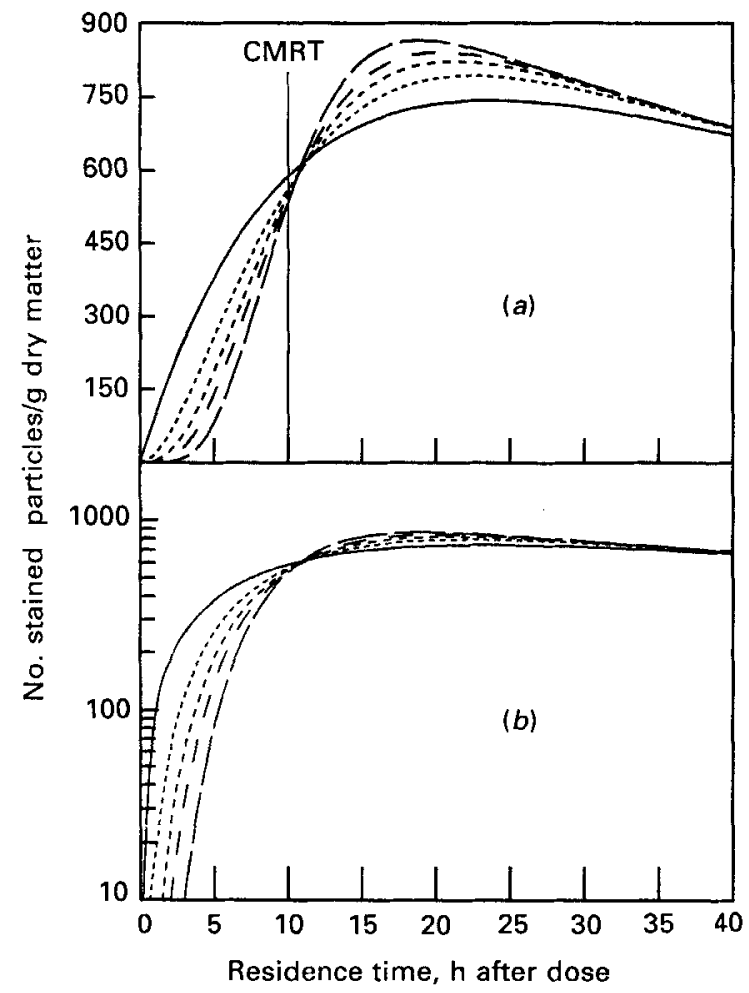

Fig. 4. The effect of increasing order of gamma distribution (G1-G4, G6) in the faster turnover compartment of a two-compartment model on the excretion pattern of particles having a compartmental mean residence time of $10 \mathrm{~h}$ in the age-dependent compartment and $80 \mathrm{~h}$ in the age-independent compartment (G1) plotted arithmetically $(a)$ and semi-logarithmically $(b)$. (- - , GlG1; (-..--),

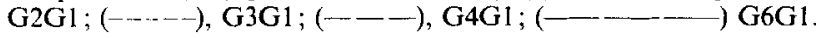

The volume of compartment 1 (in terms of similar indigestible entities as the output from compartment 2) equals $\left(D / C_{2}\right) \times\left(k_{2} / \bar{k}\right)$ where $\bar{k}$ equals $F \times \lambda_{1}$ calculated as indicated previously. The undigested volume of the two-compartment system is then the sum of the undigested volumes of its two-component compartments.

The effect of incorporating various order of age dependency (gamma distribution) in the faster turnover compartment of a two-compartment model is illustrated in Fig. 4. In this illustration, the mean compartmental residence time (CMRT) is fixed as $10 \mathrm{~h}$ for the agedependent compartment 1 (CMRT1), $80 \mathrm{~h}$ for age-independent compartment 2 (CMRT2) or $90 \mathrm{~h}$ for CMRT of the system composed of two combined compartments (CMRTS). Increasing order of gamma distribution in the faster turnover compartment results in a progressive retardation in the emergence of particles from compartment 1 as indicated earlier for an age-dependent compartment (Fig. 3). Consequently, flow from the first to the second compartment and its emergence in the output from the two-compartment system is also progressively retarded as indicated in Fig. 4. These effects of different levels of age dependency in the faster turnover compartment are proportionally diminished with progressively longer residence time in the total system. This is due to effects of the second, slower turnover compartment becoming increasingly dominant with longer residence time in the two-compartment system. Consequently, with the rate parameters illustrated in Fig. 4, the emergence of particles from the system beyond $40 \mathrm{~h}$ primarily represents the slower turnover compartment with its exponential distribution of residence times. 
Three-compartment models. Many researchers, including Milne et al. (1978), Jacquez (1985) and France et al. (1985), have presented and discussed the three-compartment, ageindependent model (G1G1G1). The total dose remaining in the system under this model is:

where

$$
D\left[\delta_{1} e^{-k_{1} t}+\delta_{2} e^{-k_{2} t}+\delta_{3} e^{-k_{3} t}\right],
$$

$$
\begin{aligned}
& \delta_{1}=k_{2} k_{3} /\left(k_{2}-k_{1}\right)\left(k_{3}-k_{1}\right), \\
& \delta_{2}=k_{1} k_{3} /\left(k_{1}-k_{2}\right)\left(k_{3}-k_{2}\right), \\
& \delta_{3}=k_{1} k_{2} /\left(k_{1}-k_{3}\right)\left(k_{2}-k_{3}\right) .
\end{aligned}
$$

CMRT. The mean time the dose resides in an age-independent compartment (CMRT) equals $1 / k$ since an exponential distribution of residence times is assumed. The CMRT in an age-dependent compartment with a gamma- $n$ distribution of residence time equals $n / \lambda_{1}$. For example, CMRT for G2 equals $2 / \lambda_{1}$ and for G6 equals $6 / \lambda_{1}$.

Time delay. Flow processess considered thus far have been compartmental effects due to mixing of particles of all resident ages. This turnover, or dilution replacement of the dose, is due to a continued input into and output from the mixing compartment(s). In contrast to compartmental turnover, flow through certain segments of the gastrointestinal tract may occur by non-mixing displacement. The term time delay, signified by tau $(\tau)$, has been proposed by Blaxter et al. (1956) to symbolize this displacement process. Since some marker instantly exits an instantaneously mixed compartment (see Gl for Fig. 3), time delay can be defined as the difference in time between dosing of the marker and its first appearance in the faeces. The parameter $\tau$ can be inserted into any model in Tables $1-4$ by substituting the expression $(t-\tau)$ for $t$ if only one time delay is assumed and estimated for the entire gastrointestinal tract.

Gastrointestinal mean residence time. The mean gastrointestinal residence time due to mixing, dilution and replacement turnover flow (CMRT) and to non-mixing displacement flow $(\tau)$ is the sum of CMRT $+\tau$, or GMRT. For a given set of data, the estimated GMRT should be approximately the same numerically for all reasonably representative models. Models will differ appreciably only in how they partition this GMRT.

\section{Animal data}

Four non-lactating Friesian cows weighing about $600 \mathrm{~kg}$ were used. Each was provided with a rumen cannula and a simple cannula in the proximal duodenum a few centimeters distal to the pylorus. (Austen et al. 1977). They were given $3.4 \mathrm{~kg}$ concentrates and $3.1 \mathrm{~kg}$ alkali-treated, spring barley straw on a dry matter (DM) basis in one meal daily.

The straw was from a single batch and was split into two halves. Half (C) was coarsely chopped to give particles averaging about $20-70 \mathrm{~mm}$ long and was then sprayed with a sodium hydroxide solution to give a concentration of about $50 \mathrm{~g} \mathrm{NaOH} / \mathrm{kg}$ straw. Half (V) was prepared by an industrial process. It was coarsely ground through a $10 \mathrm{~mm}$ screen, treated with a similar amount of $\mathrm{NaOH}$ and pelleted (Viton; Unitrition International Ltd, Basingstoke). Concentrates were a mixture of barley and a commercial protein supplement with a final crude protein (nitrogen $\times 6.25$ ) concentration of $180 \mathrm{~g} / \mathrm{kg} \mathrm{DM}$. Each cow was given both forms of straw in a single change-over design with 6-week periods.

To measure rate of passage, a portion of each type of straw was stained with magenta (Balch, 1950) and a meal containing the strained straw was given to each animal. Spot samples of duodenal digesta were obtained every $4 \mathrm{~h}$ for the first $60 \mathrm{~h}$, every $12 \mathrm{~h}$ for the next $180 \mathrm{~h}$ and every $24 \mathrm{~h}$ for a further $144 \mathrm{~h}$. Faecal samples were taken over a similar period but omitting the first $8 \mathrm{~h}$; they were obtained by mixing and sampling all the faeces that had accumulated on the floor in the interval between each sampling time. The number 
Table 5. Time delay $(\tau, h)$ and compartmental residence times $(C M R T, h)$ estimated by the model yielding the lowest error mean square (EMS) when fitted to faecal values

\begin{tabular}{|c|c|c|c|c|c|c|c|c|c|}
\hline \multirow[b]{2}{*}{ Diet } & \multirow[b]{2}{*}{ Cow no. } & \multicolumn{4}{|c|}{ Two-compartment models } & \multicolumn{4}{|c|}{ One-compartment models } \\
\hline & & Model* & EMS & $\tau$ & CMRT $†$ & Model $\ddagger$ & EMS & $\tau$ & CMRT§ \\
\hline $\mathrm{C}$ & 6 & G2G1 & 6735 & $6 \cdot 7$ & 1060 & G3 & 6735 & $6 \cdot 7$ & $106 \cdot 0$ \\
\hline $\mathrm{C}$ & 7 & G3G1II & $4705 \pi$ & 6.6 & 83.8 & G4 & 4405 & $7 \cdot 1$ & 82.9 \\
\hline $\mathrm{C}$ & 8 & $\mathrm{G} 2 \mathrm{Gl}$ & 5793 & $9 \cdot 5$ & $89 \cdot 0$ & G3 & 5793 & 9.5 & $89 \cdot 0$ \\
\hline $\mathrm{C}$ & 9 & G2G1 & 3437 & $5 \cdot 4$ & 123.5 & G3 & 3437 & $5 \cdot 4$ & $123 \cdot 5$ \\
\hline V & 6 & G6G1 & 2984 & $13 \cdot 2$ & $65 \cdot 8$ & $\mathrm{G} 2$ & 4147 & $20 \cdot 7$ & $48 \cdot 0$ \\
\hline $\mathrm{V}$ & 7 & G6G1 & 2714 & $1 \cdot 1$ & 65.9 & G4 & 3324 & 6.2 & 536 \\
\hline V & 8 & G6Gl & 4854 & 8.8 & 74.5 & G2 & 5293 & 19.0 & $54 \cdot 3$ \\
\hline V & 9 & G6G1 & 1750 & 6.8 & 80.0 & $\mathrm{G} 2$ & 3905 & 14.5 & 54.5 \\
\hline
\end{tabular}

* Model identification designates order of age dependency in the first and second flow compartments respectively.

$\dagger$ Compartmental mean residence time in the two compartments collectively.

$\ddagger$ Model identification designates order of age dependency.

$\S$ Compartmental mean residence time in the age-dependent compartment.

$\|$ Rate parameter $\lambda_{1} \simeq$ rate parameter $k_{2}$.

I A slightly lower EMS (4660) was yielded by model G4G1, see Table 6.

of stained particles in digesta or faeces was counted and the number per $g$ DM (concentration) was calculated as described by Balch (1950).

Model fitting and statistics. The non-linear, least-squares, iterative procedure of the Statistical Analysis System (SAS, 1979) was used to fit the models to the data. A gridsearch option was used to allow the program to select a best-fitting set of parameters from a wide array of possible parameter estimates. The best-fitting parameters were then subsequently used as initial-value parameters in the iterative procedure. These type models can also be fitted to data with a microcomputer program (PCNONLIN, 1985), which offers some advantages over the Statistical Analysis System in that error estimations can be obtained for secondary parameters such as CMRT which, in the present case, were derived from the primary parameters listed for the model.

To determine model goodness of fit the error mean squares (EMS) for each model were compared. To test the precision of parameter estimates, the asymptotic standard errors of the estimates were compared.

\section{RESULTS}

Faecal values

Fit of models. The model yielding the lowest error mean square and some of the estimated parameters are summarized in Table 5.

The two-compartment models with G2 or G3 order of age dependency (G2G1 or G3G1) provided the best fit to values from diet $\mathrm{C}$ while the highest order of age-dependency utilized (G6G1) was required to minimize EMS for faecal values for diet V. The onecompartment models with differing orders of age dependency yielded EMS equal to that of two-compartment models with differing orders of age dependency when fitted to faecal values from cows receiving diet $\mathrm{C}$. Where equal EMS occurred for one- and twocompartment models, the parameters and CMRT were also equal between models. Also when equal EMS occurred, the best fitting one-compartment model had an order of age dependency $(\mathrm{G} n)$ in which $n$ equalled the sum $n+1$ in the best fitting two-compartment 


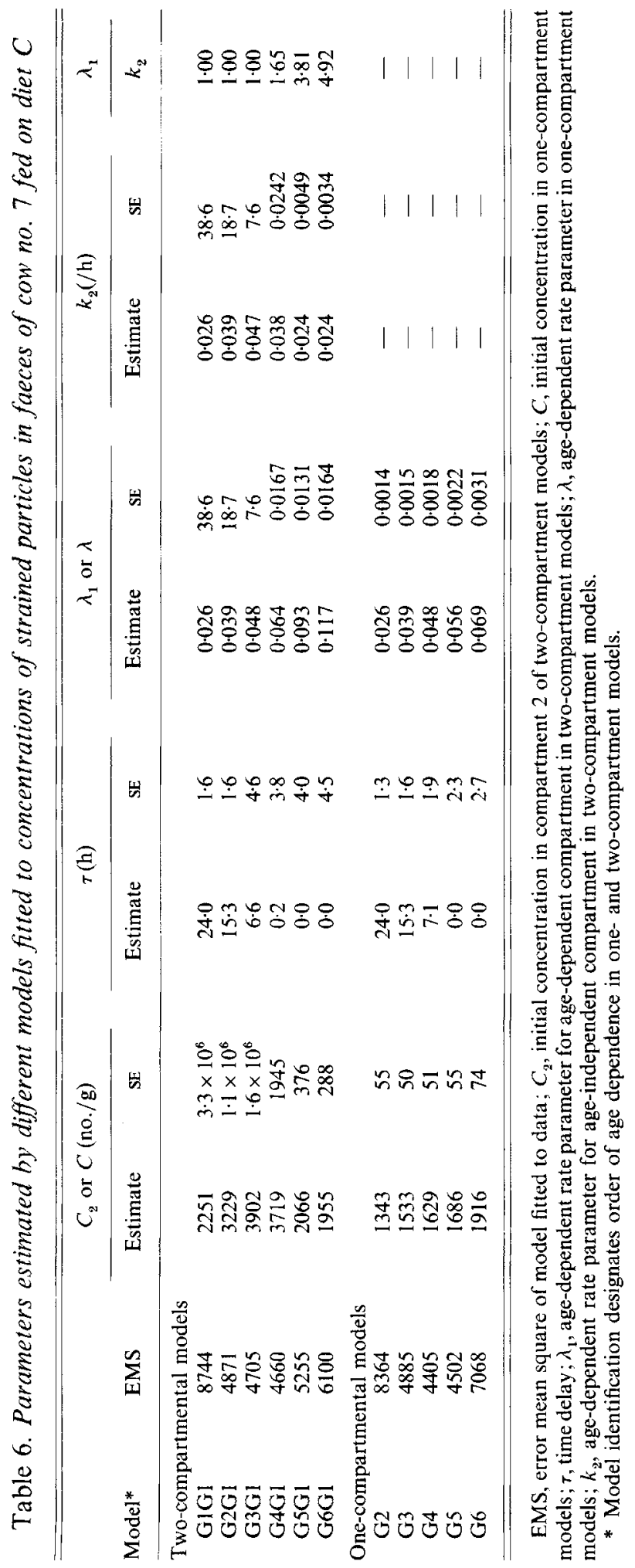


model (GnGl). When two-compartment models were fitted to faecal values from cows receiving diet $\mathrm{C}$, the G1G1 model yielded rate parameter estimates which were essentially equal. Increasing the order of age dependency in the age-dependent-turnover compartment to $\mathrm{G} 2$ resulted in a reduction of EMS and a resolution of the two rate parameters for faecal values from all animals fed on diet $\mathrm{C}$.

Values for cow no. 7 receiving diet $\mathrm{C}$ were unique among cows and diets in that the highest order of age dependency was required in both one- and two-compartment models fitted in order to minimize EMS (Table 5).

Order of age dependency, diet $C$. Results from fitting different models to values from cow no. 7 fed on diet $C$ illustrate the effects of order of age dependency and are summarized in Table 6. Increasing the order of age dependency in both the one- and two-compartment models resulted in a reduction in EMS and $\tau$ until estimates of $\tau$ approached zero $(<0 \cdot 1$ h). The $\lambda_{1}$ and $k_{2}$ rate parameters were equal $\left(\lambda_{1} / k_{2}=1 \cdot 00\right.$, Table 6$)$ in the twocompartment models until the order of age dependency exceeded that of the G3G1 model. The asymptotic standard errors of the rate parameters, $\lambda_{1}$ and $k_{2}$, and the initial concentration parameter, $C_{2}$, estimated by the G1G1, G2G1, and G3G1 models were exceedingly large since the estimation of these parameters was dependent on the model assumption of distinct rate parameters. Increasing the order of age dependency in the agedependent turnover compartment altered the model's functional specification of the faster turnover compartment so that rate parameters of a different magnitude could be resolved for the two compartments. The order of age dependency had to be increased to G5 in this particular data set before sufficiently wide differences occurred between $\lambda_{1}$ and $k_{2}$ (mean ratio of 3.81 , Table 6 ) to minimize the standard errors of the slower rate parameter $\left(k_{2}\right)$ and the concentration parameter for the slower turnover compartment, $C_{2}$. Typically, as $\lambda_{1}$ approximates $k_{2}, k_{2}$ and $C_{2}$ are systematically overestimated.

Also, increasing order of age dependency via a gamma distribution resulted in a progressive increase in the compartmental residence time attributed to the faster turnover compartment at the expense of the time-delay function, $\tau$. When $\tau$ was reduced to zero by increasing order of age dependency, no further residence time was then available and this resulted in increases in EMS when further, higher orders of gamma functions were used.

Increasing the order of age dependency in the one-compartment models fitted to faecal values from cow no. 7 fed on diet $C$ (Table 6) resulted in a decline in EMS for onecompartment models up to and including G4; after which EMS increased. The EMS and the standard error for the rate parameter, $\lambda$, and the initial concentration in the compartment, $C$, were not materially affected by the order of age dependency in the onecompartment models and were much lower than those for two-compartment models. The lower standard error for parameters estimated by the one-compartment model was not only the result of simply fewer parameters in the one-compartment model but also reflects the lack of precision in resolving residence time between two compartments when the parameters estimates are nearly equal for the two compartments.

The mean residence time partitioned by each model fitted to faecal values from cow no. 7 fed on diet $\mathrm{C}$ is summarized in Table 7 . These results indicate that any two-compartment model yielding equal rate parameters $\left(\lambda_{1}\right.$ and $\left.k_{2}\right)$ also yields estimates of $\lambda_{1}, \tau$ and CMRT which are equal to $\lambda, \tau$ and CMRT estimated by the corresponding one-compartment model. Thus when $\lambda_{1}=k_{2}$, corresponding one- and two-compartment models $(\mathrm{G} n+1=$ $\mathrm{G} n \mathrm{Gl})$ exist until the incorporation of increasing orders of age dependency $(\mathrm{G} n)$ in the faster turnover compartment of the two-compartment model results in $\lambda_{1}>k_{2}$. The EMS is minimized at the order of age dependency required for minimal resolution of $\lambda_{1}$ from $k_{2}\left(\mathrm{G} 4 \mathrm{Gl}\right.$ for these values where $\lambda_{1}=1.65 k_{2}$, Table 6). 
Table 7. Compartmental mean residence times $(C M R T, h)$ computed from rate parameters estimated from fitting different models to faecal data for cow no. 7 fed on diet $C$

\begin{tabular}{|c|c|c|c|c|c|c|c|}
\hline \multirow[b]{2}{*}{ Model* } & \multicolumn{2}{|c|}{ Rate parameter } & \multicolumn{3}{|c|}{ CMRT } & \multirow[b]{2}{*}{$\tau$} & \multirow[b]{2}{*}{ GMRT } \\
\hline & $\lambda_{1}$ or $\lambda$ & $k_{2}$ & $\begin{array}{c}\text { CMRTl } \\
\left(n / \lambda_{1}\right)\end{array}$ & $\begin{array}{c}\text { CMRT2 } \\
\left(\mathrm{l} / k_{2}\right)\end{array}$ & CMRTS & & \\
\hline $\begin{array}{l}\text { G1G1 } \\
\text { G2 }\end{array}$ & $\begin{array}{l}0.02626 \\
0.02626\end{array}$ & $\begin{array}{c}0.02626 \\
-\end{array}$ & $\begin{array}{l}38 \cdot 1 \\
76 \cdot 2\end{array}$ & $38 \cdot 1$ & $\begin{array}{l}76 \cdot 2 \\
76 \cdot 2\end{array}$ & $\begin{array}{l}21 \cdot 0 \\
24 \cdot 0\end{array}$ & $\begin{array}{l}100 \cdot 2 \\
100 \cdot 2\end{array}$ \\
\hline $\begin{array}{l}\text { G2G1 } \\
\text { G3 }\end{array}$ & $\begin{array}{l}0.03863 \\
0.03863\end{array}$ & 0.03863 & $\begin{array}{l}51 \cdot 8 \\
77 \cdot 7\end{array}$ & $\begin{array}{c}259 \\
-\end{array}$ & $\begin{array}{l}77 \cdot 7 \\
77 \cdot 7\end{array}$ & $\begin{array}{l}15 \cdot 3 \\
15 \cdot 3\end{array}$ & $\begin{array}{l}93 \cdot 0 \\
93 \cdot 0\end{array}$ \\
\hline $\begin{array}{l}\text { G3G1 } \\
\text { G4 }\end{array}$ & $\begin{array}{l}0.04774 \\
0.04823\end{array}$ & $\begin{array}{c}0.04774 \\
-\end{array}$ & $\begin{array}{l}62 \cdot 8 \\
82 \cdot 9\end{array}$ & $\begin{array}{c}21 \cdot 0 \\
-\end{array}$ & $\begin{array}{l}83 \cdot 8 \\
82 \cdot 9\end{array}$ & $\begin{array}{l}6 \cdot 6 \\
7 \cdot 1\end{array}$ & $\begin{array}{l}90 \cdot 4 \\
90 \cdot 4\end{array}$ \\
\hline $\begin{array}{l}\text { G4Gl } \\
\text { G5 }\end{array}$ & $\begin{array}{l}0.06359 \\
0.05634\end{array}$ & $\begin{array}{c}0.03851 \\
-\end{array}$ & $\begin{array}{l}62 \cdot 9 \\
85 \cdot 3\end{array}$ & $\begin{array}{c}26 \cdot 0 \\
-\end{array}$ & $\begin{array}{l}88 \cdot 9 \\
85 \cdot 3\end{array}$ & $\begin{array}{l}0.2 \\
0 \cdot 0\end{array}$ & $\begin{array}{l}89 \cdot 1 \\
85 \cdot 3\end{array}$ \\
\hline $\begin{array}{l}\text { G5G1 } \\
\text { G6 }\end{array}$ & $\begin{array}{l}0.09341 \\
0.06926\end{array}$ & $\begin{array}{c}0.02454 \\
-\end{array}$ & $\begin{array}{l}53 \cdot 5 \\
85 \cdot 3\end{array}$ & $\begin{array}{c}40 \cdot 8 \\
-\end{array}$ & $\begin{array}{l}94 \cdot 3 \\
85 \cdot 3\end{array}$ & $\begin{array}{l}0-0 \\
0-0\end{array}$ & $\begin{array}{l}94 \cdot 3 \\
86 \cdot 6\end{array}$ \\
\hline G6G1 & $0 \cdot 11714$ & 0.02385 & $55 \cdot 3$ & $33 \cdot 7$ & 89.0 & 0.0 & $89 \cdot 0$ \\
\hline
\end{tabular}

$\lambda_{1}$, Age-dependent rate parameter for the age-dependent compartment of two-compartment models; $\lambda$, agedependent rate parameter for the age-dependent, one-compartment models; $k_{2}$, rate parameter for the ageindependent compartment of two-compartment models; $n$, integer order of the gamma distribution in the agedependent compartment; CMRT, compartmental mean residence time in the two collective compartments or in the one-compartment model; $\tau$, time delay; GMRT, CMRT $+\tau$.

* Model identification designates order of age dependence in one- and two-compartment models.

The fit of some of the models to the concentrations of stained particles appearing in the faeces of cow no. 7 fed on diet $\mathrm{C}$ is shown in Fig. 5. The correspondence of $\mathrm{G} 1 \mathrm{G} 1$ to $\mathrm{G} 2$, G2G1 to G3 and G3G1 to G4 models is illustrated by the superimposability of the predicted curves from these corresponding models fitted to the same data. The G1G1 or G2 models clearly overestimated $\tau$ since detectable concentrations of stained particles were observed in the faeces before that estimated for $\tau$ (Fig. 5(a)). The incorporation of progressively higher orders of age dependence up to G4 resulted in improved fit to observed results throughout the range of observed residence times. Further increases in age dependency beyond G4 resulted in a reduced fit by the model to the observed values due to a more accentuated and delayed peak in concentration and a more rapid subsequent decline in concentration of stained particles.

Order of age dependency, diet $V$. Results of fitting various models to faecal values from cows receiving diet $\mathrm{V}$ are typified by results for cow no. 9 summarized in Table 8 . The excretion pattern of stained particles resulted in a wide separation of the rate parameters by the two-compartment, age-independent model (GlGl). Increasing the order of age dependency in the faster turnover compartment of the two-compartment model resulted in progressive reductions in EMS although such reductions became relatively small for orders of age dependency beyond G4G1. The one-compartment models all yielded EMS considerably greater than those for the two-compartment models.

The fit of predicted values for the G1G1 and G6G1 models and the observed values for cow no. 9 fed on diet $V$ are shown in Fig. 6. The age-independent, exponential distribution of particles having residence times beyond $50-60 \mathrm{~h}$ is clearly evident from the linear nature of the semi-logarithmic plot (Fig. 6(b)). Improvements in fit as the result of increasing order of age dependency in the faster turnover compartment were largely due to a better fit for particles having residence times of less than $30 \mathrm{~h}$, as indicated in Fig. $6(\mathrm{c})$. Clearly, the age- 


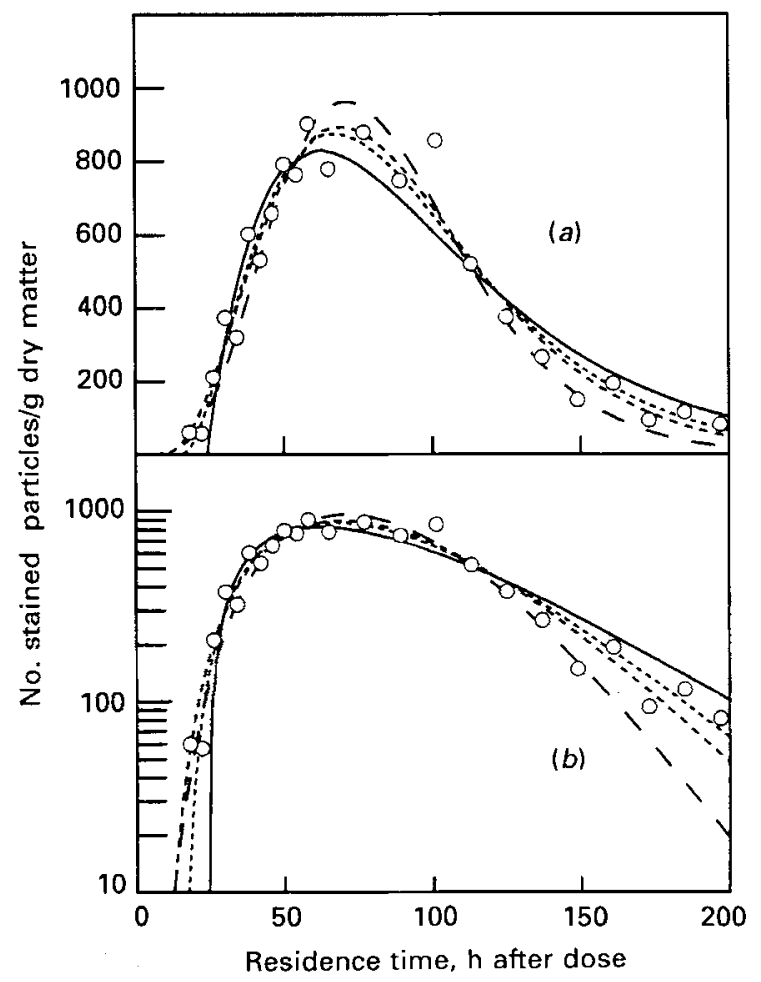

Fig. 5. Observed results $(O)$ and predicted curves for various models fitted to faecal values for cow no. 7 fed on diet C plotted arithmetically $(a)$ and semi-logarithmically $(b)$. ( -$)$, GIG1 and G2; (-- - -),

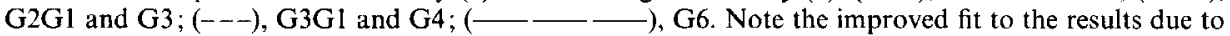
progressively increasing the order of age dependency in both one- and two-compartment models. Also note the superimposability of two-compartment models yielding equal rate parameters with onecompartment models having an order of gamma distribution equal to the sum of the orders of gamma distributions in the two-compartment model with equal rate parameters; i.e. (a) G1Gl and G2, (b) G2Gl and G3, and (c) G3GI and G4, are corresponding two- and one-compartment models and therefore superimposable.

independent, two-compartment model (G1G1) overestimated $\tau$ since this estimate $(16.7 \mathrm{~h})$ was greater than the time of first observable stained particle $(14 \mathrm{~h}$ mid-point of a $12-16 \mathrm{~h}$ faecal collection interval). Thus, estimates of $\tau$ as low as $6.8 \mathrm{~h}$, as predicted by the G6G1 model, appear feasible for the first detectable appearance of marker in the faeces.

Effect of diet. The forms of the observed and predicted excretion patterns in the faeces for cow no. 9 fed on diet $\mathrm{V}$ or diet $\mathrm{C}$ are compared in Fig. 7. The excretion pattern of particles from diet $\mathrm{V}$ was dominated by an exponential distribution of residence times during the last 250 of the $300 \mathrm{~h}$ of observable stained particles. In contrast, the excretion pattern of particles from diet $\mathrm{C}$ was much delayed in achieving peak concentration and thereafter decreased at an ever-increasing rate without clear evidence of ever attaining an exponential distribution of residence times (linear decline on a semi-logarithmic plot).

Number of compartments. The excretion pattern of stained particles by certain cows (see Fig. 8) suggested the contribution of a third compartment. Results of fitting a threecompartment model with exponentially distributed residence times to values for diets $\mathrm{V}$ and $\mathrm{C}$ are summarized in Table 9. For animals fed on diet $\mathrm{V}$, the three-compartment model yielded estimates for two rates of a similar magnitude and a second, slower, third rate. The slower rate was of similar magnitude to the slower, age-dependent rate estimated by the 


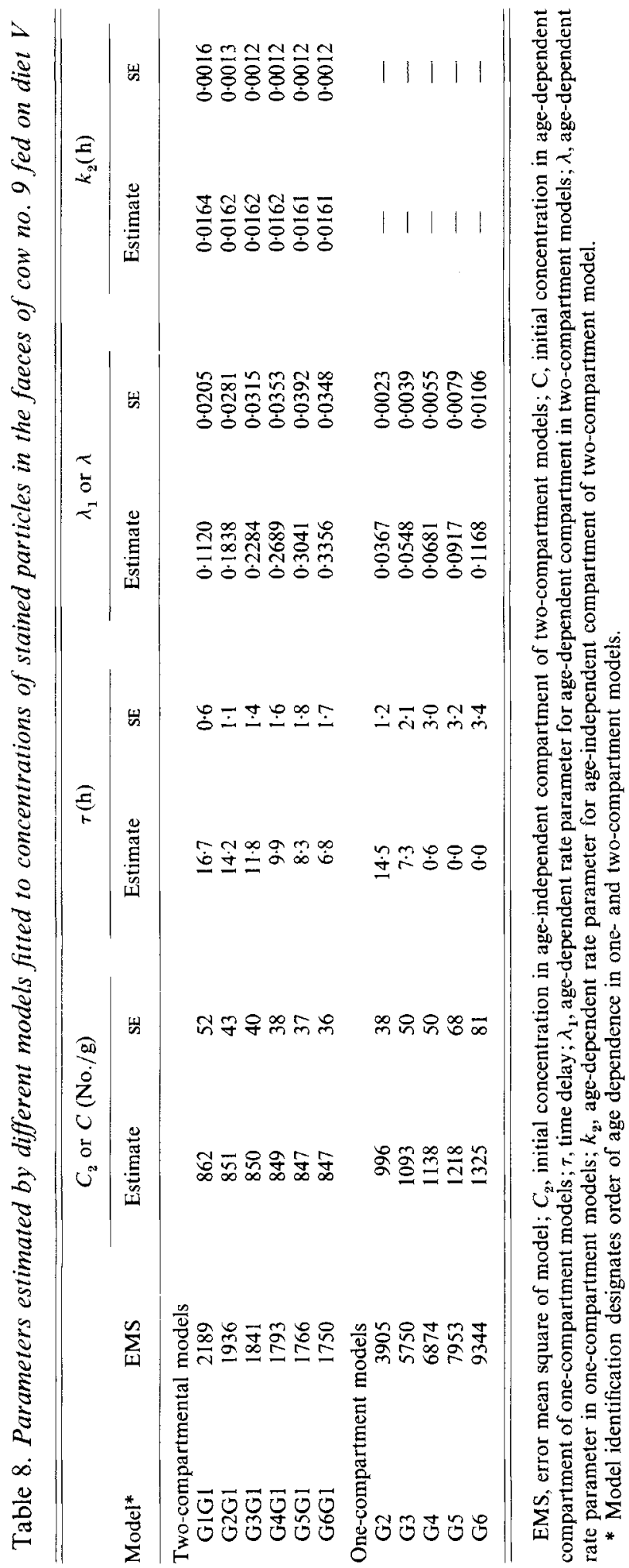




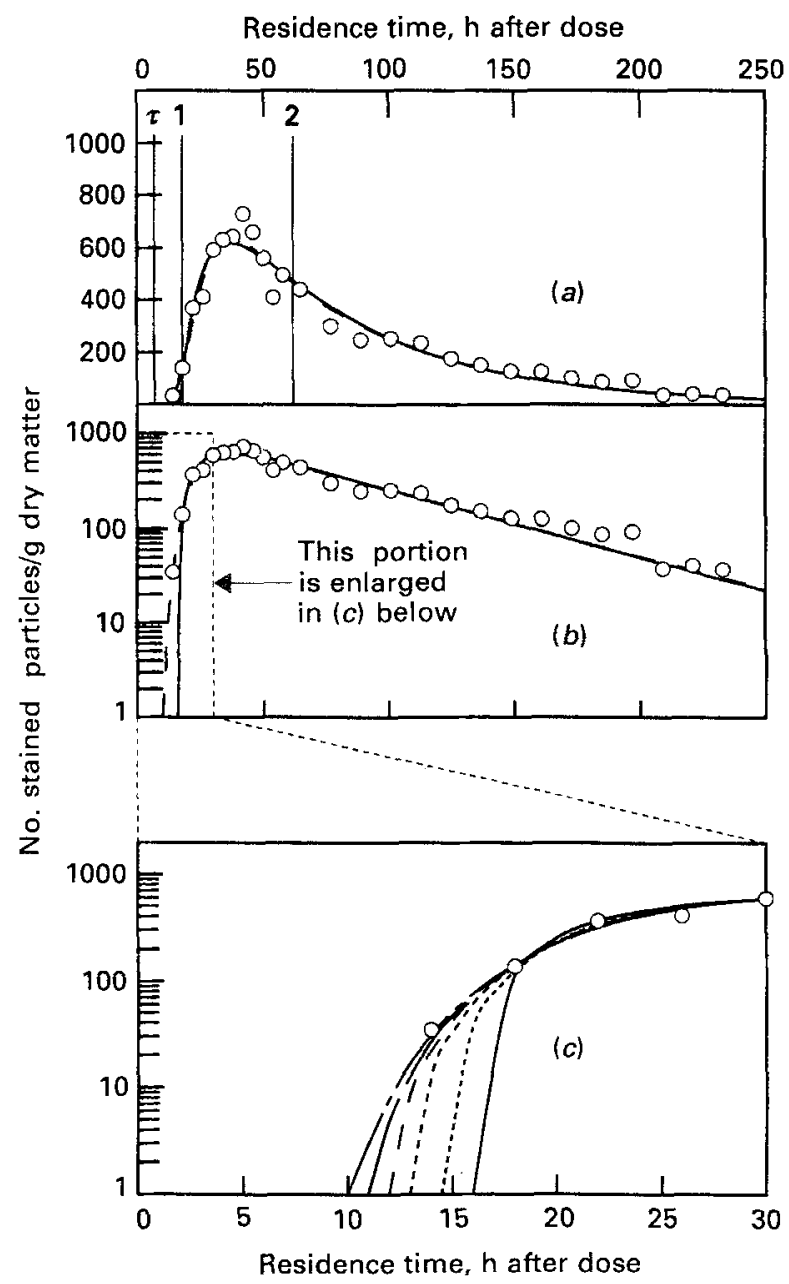

Fig. 6. Observed results $(O)$ and predicted curves for various two-compartment models fitted to faecal values for cow no. 9 fed on diet V. In $(a)$ the time delay $(\tau)$ and compartmental mean retention time in the age-dependent (1) and age-independent (2) compartments are indicated arithmetically for models GIG1 ( $\longrightarrow$ and G6G1 (---). In $(b)$ the same results and model curves are presented semilogarithmically. In $(b)$, note the dominance of exponential distribution (Gl) of the longer residence times $(>30 \mathrm{~h}$ ) and the lack of difference in longer-residence-time distributions predicted by either the GlGl or G6G1 models in the semi-logarithmic graph. The effect of various orders of gamma distribution (G1-G6) on fit for the faster turnover compartment to the earliest observed results for stained particles appearing in the faeces (shorter residence times) is illustrated in $(c)$. In $(c)$, note the progressive increases in fit to the first-observed result as a consequence of increasing orders of gamma distribution from Gl to $\mathrm{G} 6$ and its effects on reducing estimates of time delay for first appearance of stained particle in faeces. Predicted curves in $(c)$ are for models GlGl (-), G2G1 (-....), G3G1 (-- ), G4Gl (- - -), G5G1 (

age-independent (G1) compartment of the best fitting two-compartment, age-dependent model. It appears that the three-compartment, age-independent model (G1GlG1) attempted to resolve different rates from the faster turnover portion of the residence-time distribution rather than the slow turnover portion suspected to reflect the third compartment (Fig. 8). 


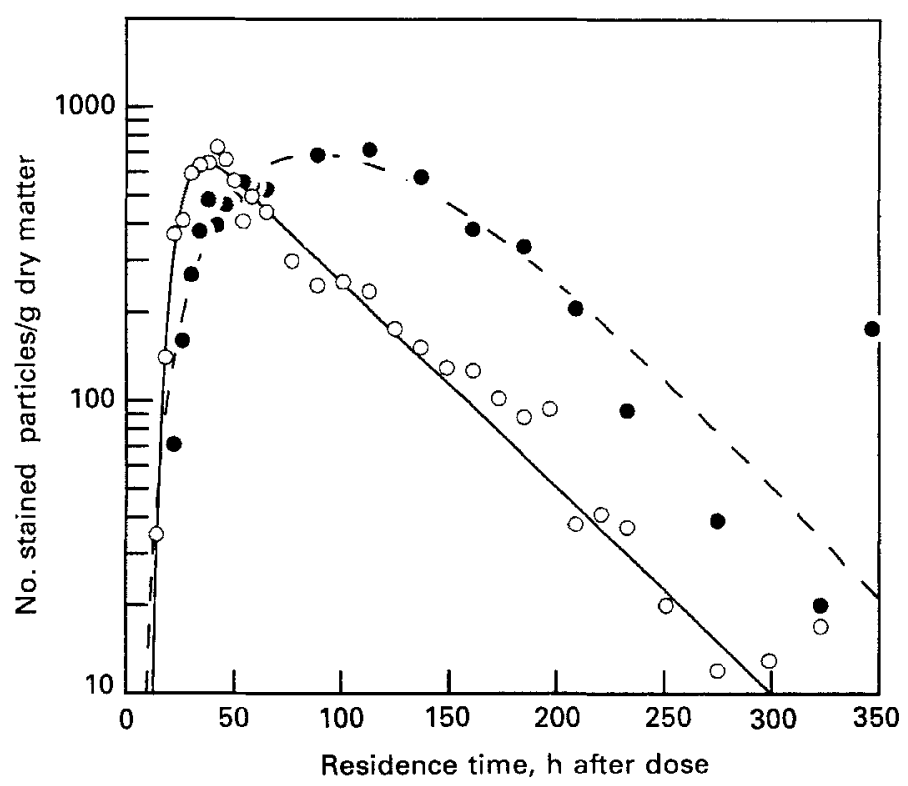

Fig. 7. Comparison of observed results for cow no. 9 fed on diet $\mathrm{V}(\mathrm{O})$ or diet $\mathrm{C}(\mathrm{O})$ and predicted curves for model G6Gl (_- ) and model G3 (- - - Note the distinctly different, bi-phasic distribution of residence times exhibited for diet $V$ and the dominance of exponentially distributed (G1) residence times as evidenced by the linear phase of the semi-logarithmic plot. In contrast, the distribution of residence times of stained particles from diet $\mathrm{C}$ is delayed in general, the exponential distribution of residence times is much delayed and less dominating, and the overall distribution appears the result of a continuum of ever-decreasing rates of turnover.

When applied to the faeces of cows receiving diet $\mathrm{C}$, the three-compartment model yielded two slower turnover rates of similar magnitude to each other and to that of the mean age-dependent rate $\left(\lambda_{1} \times F\right)$ and $k_{2}$ estimated by the best fitting two-compartment, age-dependent models. Thus, the three-compartment model did not respond to the excretion pattern suggesting a third, slower turnover compartment.

Time delay. Dietary means are summarized in Table 10 for the $\tau$ estimated by a number of different models. It has been noted (Figs 5 and 6 ) that the G1Gl model commonly yields estimates for $\tau$ which are later than the first-observed occurrence of stained particles in the faeces. The exponentially distributed three-compartment model (G1G1G1) yielded estimates of $\tau$ of a similar magnitude to that of the G1G1 model. Appreciably lower estimates of $\tau$ resulted from the inclusion of age dependency at a level to provide best fitting of two-compartment models to faecal values from cows fed on diet V (G6Gl) and onecompartment models to provide best fitting of faecal values from cows fed on diet C (G3 and G4).

One-compartment, age-dependent models yielded similar mean estimates of $\tau$ for the four cows fed on either diet $\mathrm{C}$ or diet $\mathrm{V}(7.2$ and $7.0 \mathrm{~h}$ respectively). These mean estimates were of a similar magnitude to that estimated by the G6G1 model for the four cows fed on diet $\mathrm{C}(7.5 \mathrm{~h})$. However, the G6G1 model yielded estimates of 0.0 for the $\tau$ for all cows fed on diet C. This estimate of 0.0 for $\tau$ was due to a lack of strategically critical values during the early excretion periods from cows fed on diet $\mathrm{C}$ to accurately partition CMRT1 from $\tau$, and is discussed in detail later. 


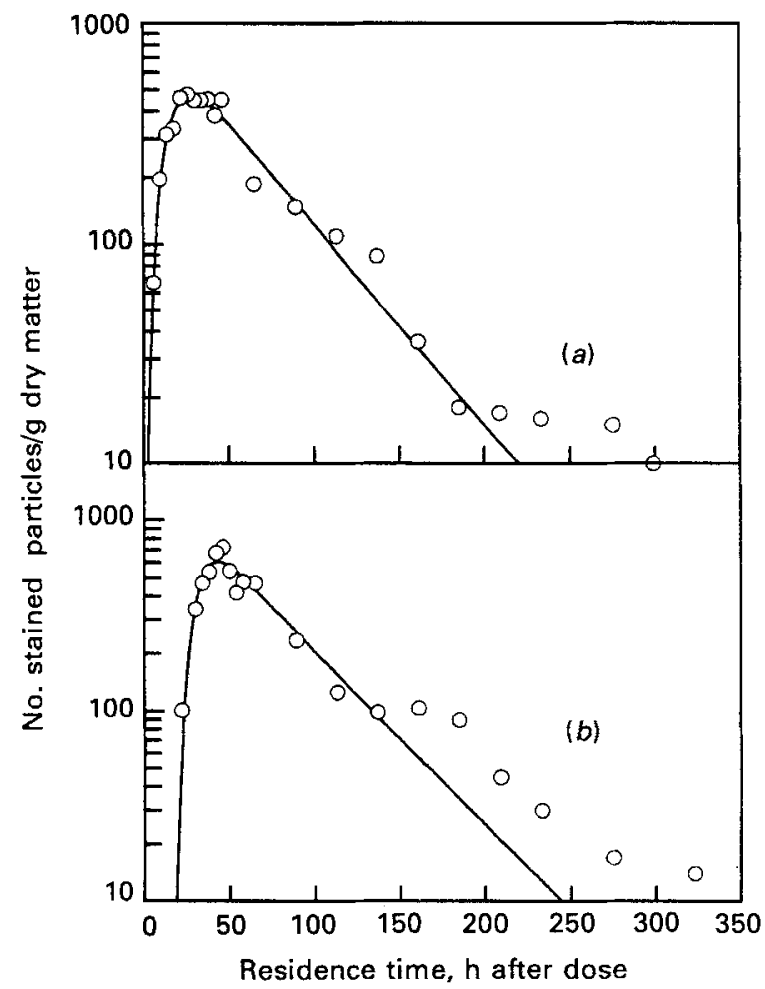

Fig. 8. Observed results $(O)$ and predicted curves for stained particles appearing at the duodenum $(a)$ and faeces $(b)$ of cow no. 6 fed on diet V. Predicted curve is for model G2Gl fitted to duodenal values $(a)$ and for model G6G1 fitted to faecal values $(b)$. Note the deviations from the predicted curves of longer-residence-time particles which suggest the occurrence of a third compartment of slower turnover than the exponentially distributed (Gl) compartment of the two-compartment models. Also note the superimposability of the predicted curves for duodenal $(a)$ and faecal $(b)$ sites. The compartmental mean residence times for the age-dependent and age-independent compartments respectively, was 16.6 and $46.9 \mathrm{~h}$ at the duodenum and 8.7 and $47.1 \mathrm{~h}$ at the faeces; a numerical similarity which expresses superimposability of excretion pattern at these two sites for this cow fed on diet $\mathrm{V}$.

Table 9. Dietary means for error mean square (EMS) and rate parameters $(/ h)$ estimated by models* having three exponentially distributed residence compartments (G1GIGI) or a gamma distributed-exponentially distributed, two-compartment model (G3G1, G4G1)

(Mean values for four cows fed on each diet)

\begin{tabular}{|c|c|c|c|c|c|c|c|}
\hline \multirow[b]{2}{*}{ Diet } & \multicolumn{4}{|c|}{ GlGlGl } & \multicolumn{3}{|c|}{ G3G1, G4G1† } \\
\hline & EMS & $k_{1}$ & $k_{2}$ & $k_{3}$ & EMS & $\lambda_{1} \times F$ & $k_{2}$ \\
\hline $\mathrm{C}$ & 7079 & 0.203 & 0.023 & 0.021 & 5709 & 0.023 & 0.022 \\
\hline V & 3542 & $0 \cdot 119$ & $0 \cdot 118$ & 0.022 & 3264 & 0.087 & $0 \cdot 022$ \\
\hline
\end{tabular}

$k_{1}, k_{2}, k_{3}$, rate parameters for exponentially distributed residence times in compartments 1,2 and 3 of GlGlGl model; $\lambda_{1}$, rate parameter for age-dependent compartment of G3G4 or G4G1 models; $F$, constant to convert $\lambda_{1}$ to mean rate; $k_{2}$, rate parameter for age-independent compartment of G3G1 or G4G1 models.

* Model identification designates order of age dependence in one- and two-compartment models.

$\dagger$ Cow no. 7 
Table 10. Dietary means for time delay $(h)$ estimated by different models

(Mean values with their standard errors for four animals per diet)

\begin{tabular}{|c|c|c|c|c|c|c|c|c|}
\hline \multirow[b]{3}{*}{ Diet } & \multicolumn{8}{|c|}{ Model* } \\
\hline & \multicolumn{2}{|c|}{ G1G1 } & \multicolumn{2}{|c|}{ GlGiGl } & \multicolumn{2}{|c|}{ G6Gl } & \multicolumn{2}{|c|}{ G3, G4† } \\
\hline & Mean & SE & Mean & $\mathrm{SE}$ & Mean & SE & Mean & SE \\
\hline $\mathrm{C}$ & $20 \cdot 8$ & 1.8 & 17.7 & $1 \cdot 8$ & 0.0 & - & $7 \cdot 2$ & 1.5 \\
\hline V & $19 \cdot 1$ & 1.7 & $19 \cdot 2$ & 1.6 & 7.5 & $4 \cdot 3$ & 7.0 & $2 \cdot 8$ \\
\hline
\end{tabular}

* Model identification designates order of age dependency in compartments.

$\dagger$ G4 for Cow no. 7, G3 for others.

Table 11. Parameters estimated by the model yielding the lowest or next to the lowest error mean square (EMS) when fitted to duodenal values

\begin{tabular}{|c|c|c|c|c|c|c|c|c|c|c|}
\hline \multirow[b]{2}{*}{ Diet } & \multirow[b]{2}{*}{ Cow no. } & \multicolumn{4}{|c|}{ Two-compartment models } & \multicolumn{5}{|c|}{ One-compartment models } \\
\hline & & Model* & EMS & $\tau$ & CMRT $\dagger$ & & Model $\ddagger$ & EMS & $\tau$ & CMRT§ \\
\hline $\mathrm{C}$ & 6 & GlGl & 15066 & $2 \cdot 5$ & $93 \cdot 6$ & & G2 & 15078 & $2 \cdot 3$ & $92 \cdot 4$ \\
\hline C & 7 & $\mathrm{G} \mid \mathrm{G} 1 \|$ & 14753 & $3 \cdot 3$ & $80 \cdot 1$ & & $\mathrm{G} 2$ & 14753 & $3 \cdot 3$ & $80 \cdot 1$ \\
\hline $\mathrm{C}$ & 8 & $\mathrm{G} \mid \mathrm{G} I$ & 9154 & $1 \cdot 4$ & $88 \cdot 1$ & & G2 & 9154 & $1 \cdot 4$ & $88 \cdot 1$ \\
\hline $\mathrm{C}$ & 9 & GIGI & 10834 & $8 \cdot 2$ & $98 \cdot 3$ & & $\mathrm{G} 2$ & 10834 & $8 \cdot 2$ & $98 \cdot 4$ \\
\hline $\mathrm{C}$ & 9 & G2G1 & 10345 & 0.0 & 103.5 & & G3 & 11429 & $0 \cdot 0$ & $94 \cdot 0$ \\
\hline V & 6 & GlGi & 1378 & $6 \cdot 3$ & $55 \cdot 7$ & & $\mathrm{G} 2$ & 1282 & $4 \cdot 8$ & $50 \cdot 5$ \\
\hline V & 6 & $\mathrm{G} 2 \mathrm{G} 1$ & 1009 & $2 \cdot 1$ & $60 \cdot 2$ & & G3 & 1771 & 0.0 & $49 \cdot 0$ \\
\hline V & 7 & GlGl & 3374 & 3.9 & 55.9 & & $\mathrm{G} 2$ & 3347 & $2 \cdot 8$ & $49 \cdot 8$ \\
\hline $\mathrm{V}$ & 8 & GlGI & 2440 & $2 \cdot 3$ & 57.8 & & G2 & 2765 & 1.4 & $50 \cdot 6$ \\
\hline V & 9 & $\mathrm{GlGl}$ & 3216 & $3 \cdot 1$ & $75 \cdot 4$ & & $\mathrm{G} 2$ & 5685 & 0.0 & $54 \cdot 3$ \\
\hline V & 9 & $\mathrm{G} 2 \mathrm{G} 1$ & 2969 & 00 & $78 \cdot 1$ & - & - & - & - & \\
\hline
\end{tabular}

$\tau$, Time delay; CMRT, compartmental mean residence time.

* Model identification designates order of age dependency in one- and two-compartment models.

+ Compartmental mean residence time in the two compartments collectively.

¥ Model identification designates order of age dependency.

$\$$ Compartmental mean residence time in the age-dependent compartment.

$\|$ Rate parameter $\lambda_{1} \simeq$ rate parameter $k_{2}$.

\section{Duodenal values}

Fit of models. With one exception (cow no. 9 fed on diet V, Table 11), fitting twocompartment models to duodenal values yielded equal estimates for the rate parameters. Compared with faecal values, relatively lower orders of age dependency (G1G1, G2G1) were required to minimize EMS (with the exception of cow no. 9 fed on diet V). One- and two-compartment models yielded similar EMS and were generally indicated to be corresponding models by the occurrence of equal rates (with the exception of cow no. 9 fed on diet V).

Increasing order of age dependency in either the best fitting two- or one-compartment models (indicated in Table 11) resulted in increases in EMS. Increases in EMS were the result of the higher order of age dependency estimating a $\tau$ which approximated its constrained value of zero. A larger $\tau$ was estimated by G1G1 and G2 models for all animals fed on diet $\mathrm{C}$ and for cow nos. 6 and 9 fed on diet $\mathrm{V}$. In these six cases, increasing order 
of age dependency in the faster turnover compartment to G2 could be accommodated without reducing $\tau$ to zero and consequently reduced EMS (Table 11). Increasing the order of age dependency to G3 in the one-compartment models resulted in estimates of $\tau$ approaching the zero constraint for this parameter and was the cause of increased EMS for the G3 compared with the G2 model.

These results indicated that diet had a similar effect on model-fit to duodenal values as was observed for faecal values. Digesta flow to the duodenum of cows fed on diet $\mathrm{C}$ was best represented by a single-component, age-dependent model (G2, G3) while that from diet $\mathrm{V}$ was best represented by a two-compartment model (G2G1, G3G1) in which the order of age dependency was lower than that observed for faeces (G6G1).

\section{DISCUSSION}

A number of aspects of these results indicate an age-dependent process to be very descriptive of digesta flow through a portion if not all of the cow's digestive tract. These include:

1. An equal fit for a single age-dependent-distributed compartment compared with that yielded by multiple, age-independent-distributed compartment models for all cows fed on diet $\mathrm{C}$ (Table 5).

2. An age-dependent flow process was suggested by near-equal rate parameters when two- and three-compartment models with exponentially (age-independent) distributed residence times were fitted to excretion of stained residues in the duodenum and faeces of cows fed on diet $\mathrm{C}$.

3. Two-compartment models with age-dependent and age-independent compartments provided differences in structure which (a) allowed an identification of age dependency with the faster turnover compartment, (b) resolved otherwise equal rate parameters and (c) increased the precision of estimating parameters.

4. The association of age dependency specifically with the faster turnover compartment of two-compartment models fitted to faecal values for cows fed on diet $V$ resulted in an improved fit for the early excretion phases of stained residues (Table 8 and Fig. 6) and thereby yielded more realistic estimates of time delay. Time delays of $7 \cdot 1-7 \cdot 5 \mathrm{~h}$ estimated by the age-dependent models (Table 10) are similar to residence times estimated by other techniques (Warner, 1981) for the total of the more tubular segments (small intestines) of the cow's gastrointestinal tract and segments in which the digesta is relatively dry and therefore incapable of compartmental mixing (distal colon and rectum).

In addition to these statistical aspects based on alternative models, the occurrence of age dependency is clearly indicated by the mechanisms involved in flow of particulate matter through the ruminants' digestive tract. Although particle size reduction should theoretically be a first-order process and appears to approximate such (Pond et al. 1984), size per se is not the only determinant of particle outflow from digestive segments which could act as compartments. Sutherland (1988) has recently emphasized some interactions between the mixing action of reticulo-rumen motility and the 'unmixing' action of forage particles. Some age-dependent interactions involve digestive changes in particle morphology, specific gravity, buoyancy, rate and extent of fermentation, and contribution to digesta structure. The inertia associated with the mixing of at least some newly ingested particles with aged particles in the reticulo-rumen is also clearly indicated in extreme cases by the 'pseudo-rumination' phenomenon described by Deswysen (1981). Clearly, feed particles encounter numerous processess and forces within the digesta structure of the reticulo-rumen which motivate or constrain their movement and escape from the reticulo-rumen. Further, these effects change as the particle undergoes chemical and physical change, i.e. ageing. 
Functionally, an age-independent compartment may be defined as anything that determines the boundaries within which particles are instantly mixed. In this context, reticulo-rumen digesta could be viewed as containing a large number of such instantly mixing compartments having reversible flow, among many, if not all, such functional compartments. Conceptionally, a polycompartment, reversible-flow, mechanistic model might be conceived to describe such flow. However, such a reversible model with more than two compartments is difficult or impossible to solve based on outflow data from the total system or sampling from specific intermediate compartments which may lack functional definition.

The use of gamma residence-time distributions of $\mathrm{G} 2$ or more in the current models is derived from a stochastic approach to the model (Matis, 1987). A major philosophical advantage of such a stochastic approach is the recognition of uncertainty in the exact mechanisms involved. The practical advantage of stochastic models with the gamma residence times used here is their flexibility of application and ability to approximate complex processes by relatively few parameters, and thereby lead to solvable models at levels of biological interest (Matis, 1987). The flexibility of such stochasticity is demonstrated in the current results by its application to only the shorter-residence-time particles (two-compartment models, diet V) or to the entire spectrum of residence times associated with all particles (one-compartment models, diet C).

Dhanoa et al. (1985) have described a multi-compartment model involving an unlimited number of exponentially distributed residence-time compartments $(m)$ having sequential and irreversible turnover rates $(k)$. Solution of such a complex model was possible by further assuming a pattern of differences $(\Delta)$ between progressively larger $k$; thus $k_{i+1}=$ $k_{i}+\Delta$ where $i>1$. This polycompartmental model was fitted then by estimating only three parameters; $m, \Delta$ and $k_{2}$. However, systematic deviations occurred between observed and predicted values by this model, unless it was combined with an exponentially distributed residence-time compartment (compartment A, eqn (1) of Dhanoa et al. 1985). When this latter model was fitted to eighty-two sets of marker concentrations in the faeces, the results suggested that flow of marker to the faeces could be portrayed as involving ten to sixty irreversible compartments, each having exponentially distributed residence times and distinguishable by some progression in the magnitude of the turnover rates $(\Delta)$.

Based on fit to eighty-two data sets, Dhanoa et al. (1985) concluded that their multicompartment model was superior to all other models that they compared, including the G2G1 model described here. They indicated that the G2G1 model could be fitted only to forty-four of the eighty-two excretion curves (without specifying their lack-of-fit criteria). Moreover, Dhanoa et al. (1985) reported that the G2G1 model was 'very sensitive to initial data points and in many cases there was poor agreement between the predicted and observed concentrations in the ascending phase of the curve'. This observation is true for the singular use of G2G1 and reflects the need to consider orders of gamma distribution other than G2, as indeed was earlier emphasized by Matis (1972).

The importance of adequate, well-distributed data points in the early excretion times and in fitting different orders of gamma distribution in the age-dependent compartment of a two-compartment model is clearly illustrated in Fig. 6(c) of the current paper. The first few data points are, of necessity, critical in determining the faster turnover rate parameter, $\lambda_{1}$, and, therefore, partitioning residence time between time delay, $\tau$, and CMRT $\left(n / \lambda_{1}\right)$ in the faster turnover compartment. Such early data were generally lacking in the current data.

The distinction between delay due to progressively slower ageing effects in compartmental turnover flow $\left(n / \lambda_{1}\right)$ and displacement effects of non-compartmental flow $(\tau)$ becomes increasingly problematical as the order of gamma distribution increases. Increasing orders of gamma distribution in the faster turnover compartment requires progressively more 
strategic data during the earliest periods of marker excretion to estimate $\tau$ reliably, Otherwise, the lack of such data will result in estimates of $\tau$ equal to the lower bounds established for this parameter $(0 \cdot 0)$ by the iterative-fitting procedure and the allocation of all the earliest residence time to CMRT2 (see results in Table 7).

It should be noted that all compartmental models based on exponential retention times (i.e. the sums of exponential models) assume instantaneous mixing and steady-state conditions. The volume in and outflow of digesta from the reticulo-rumen is not steadystate but varies with feeding activity (Reid et al. 1979). Further, fitting of faecal values is complicated by variable time delays associated with emptying of the rectum. This variation results in deviations from steady-state assumptions which are especially manifested in the earliest emerging samples following a dose of marked particles. Collectively, these deviations are a source of error for all steady-state models; errors which can only be reduced by more frequent sampling, especially during the period of first emergence of the marker excretion.

The use of age dependency does not overcome errors due to systematic departure from steady-state assumptions. However, it does accommodate lack of instantaneous mixing which age-independent models, such as those of Dhanoa et al. (1985), will detect as additional irreversible compartments.

The use of gamma residence-time distributions to represent age dependence in twocompartment models conceives two functionally distinct compartments, one age-dependent and one age-independent. France et al. (1985) and Dhanoa et al. (1985) have narrowly (and unnecessarily) interpreted an age-dependent compartment of G2 to represent two contiguous pools having the same rate-constant. Matis (1987) has emphasized that this representation is only a mathematical convenience to represent age dependency. Matis (1987) discusses in greater detail the involvement of stochasticity in the present models and their comparison to the models of France et al. (1985) and Dhanoa et al. (1985).

Biological interpretation. The current results were obtained by sampling outflow from the total system (faeces) as well as a subset of the system (duodenum). Therefore, it is possible to allocate compartmental flow to some anatomical sites within the system. The CMRT estimated by the most appropriate models applied to duodenal and faecal values are summarized and compared in Table 12.

For the four cows fed on diet V, the CMRT in the slower turnover, age-independent compartment (CMRT2) was similar whether estimated at the duodenum or the faeces. This indicates that the pre-duodenal segments were the only anatomical sites of the slower, ageindependent turnover compartment in cows fed on diet V. In contrast, the CMRT in the faster turnover, age dependent compartment (CMRT1) estimated at the duodenum was 0.585 that for the faster turnover compartment estimated from faecal values. Thus, the faster turnover rate detected by the two-compartment models reflects compartmental mixing flow in both pre- and post-duodenal segments of cows fed on diet V. The sum of CMRT for the two compartments (CMRTS) in the pre-duodenal compartmental system was 0.88 that of the whole gastrointestinal tract of cows fed on diet V.

Although the excretion pattern of particles from diet $\mathrm{C}$ can be fitted by a twocompartment model, the $\tau$ is unrealistically estimated as zero. In cows fed on diet $\mathrm{C}$, this is due to the lack of data points in the early phases of the excretion pattern which are required for the two-compartment models to resolve residence times due to CMRT1. If the $\tau$ as estimated by the more robust G3 and G4 models (involving the entire excretion pattern data) is assumed to be a better estimate for cows fed on diet $\mathrm{C}$, this mean value $(7 \cdot 1 \mathrm{~h}$, Table 10$)$ can be deducted from CMRT1 to yield a corrected mean estimate of CMRT in the faster turnover compartment of cows fed on diet $C$.

The mean corrected estimate of CMRT1 in pre-duodenal segments of cows fed on diet $\mathrm{C}(27.6 \mathrm{~h})$ was 0.62 of that estimated for the CMRT1 detected in the faeces. This proportion 


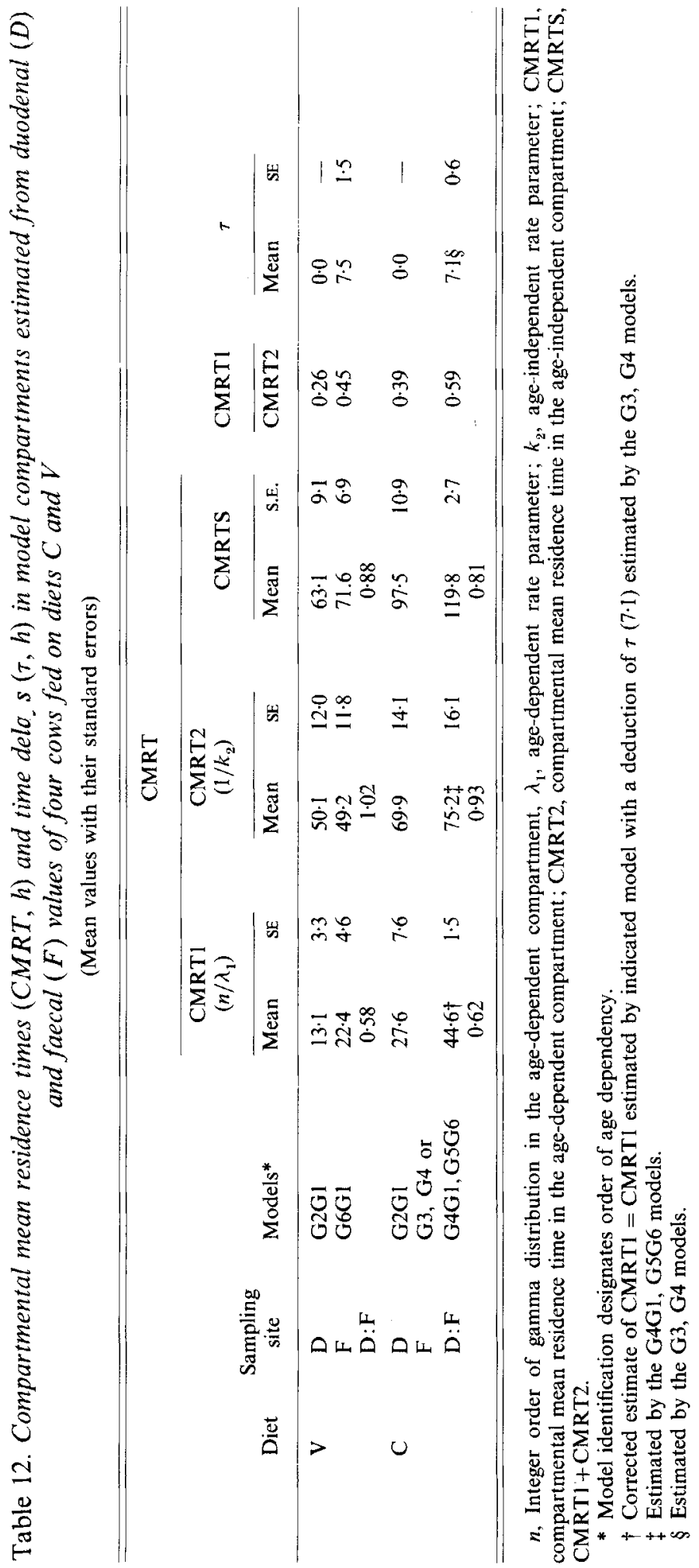


compares closely with 0.58 estimated for cows fed on diet V. As for diet $\mathrm{V}$, residence time in the slower turnover compartment (CMRT2) of cows fed on diet C appeared to be largely accounted for by pre-duodenal flow ( 0.93 and 1.00 respectively).

The results in Table 12 and interpretations as discussed here are supported by a larger number of data sets with cattle (Pond et al. 1982; Ellis et al. 1984). This interpretation of pre-duodenal sites as the dominant source of the slower compartment turnover (CMRT2) and the major source of CMRTS is, however, contrary to some observations by Faichney \& Boston (1983) and Faichney (1984) who observed comparatively longer residence times in the hind-gut of pregnant sheep than in the reticulo-rumen.

Models and marker techniques for estimating residence time of specific feed nutrients in the reticulo-rumen of non-surgically modified animals are of interest in order to partition site, extent and composition of products of ruminant digestion (Waldo et al. 1972; Broderick, 1978). For example, Ørskov \& McDonald (1979) have proposed a system for estimating degradability of dietary protein in the reticulo-rumen based on reticulo-rumen residence time estimated as an exponential distribution of residence times for the declining phase of marker excretion in the faeces. Based on the current result and other results (Pond et al. 1982; Ellis et al. 1984), this phase of faecal marker excretion does appear to represent a process confined to the reticulo-rumen. Based on results in Table 12, (CMRT1/CMRT2), an additional residence time of $0 \cdot 26-0.39$ that of the CMRT2 detected in the faeces also occurs in the reticulo-rumen. It should be noted, however, that the technique used by Ørskov \& McDonald (1979) singularly to estimate this exponentially distributed rate will produce confounded overestimates of the slower rate compared with the simultaneous estimation procedures used here.

Modelling objectives and strategy. Two-compartment models are indicated when: (1) the modelling objective is to partition total gastrointestinal compartmental residence time or, (2) when the data clearly exhibit a distribution of exponential residence times which dominate the declining segment of the excretion curve, as was observed here for cows fed on diet $\mathrm{V}$ (for example, see Fig. 7).

The recommended strategy is initially to fit G2G1 models to the data and, if necessary, then increase the order of gamma distribution to a level which resolves the two rate parameters by a factor of at least four. (The alternative of an age-dependent compartment with a non-integer-shape parameter, such as used by Roux \& Pienaar (1984) for a one-compartment model, is mathematically intractable when used with a twocompartment model.) The distribution, number and quality of data for early-emerging marker should be visually examined to ensure that the data are adequate for estimating the faster rate parameter and resolving it from $\tau$. Inadequate data in the first emerging phase of the excretion curve will result in unrealistically low estimates of $\tau$, especially if the order of gamma distribution exceeds 2 or 3 (as observed in Table 6). The statistically optimal choice of times of data collection is addressed by Landaw (1985). Experience has indicated that orders of gamma distribution from 2 to 3 have been sufficient to resolve the two rate parameters and have the greatest effect on minimizing EMS.

If the distribution and quality of the data are limited and resolution of compartments is not an objective, the more robust one-compartment models are recommended. The EMS and predicted values for one- and two-compartment models should be compared to ensure that higher orders of gamma distribution do not result in systematic deviations by the one, age-dependent models for the longer-residence-time markers such as will occur with clearly dominant exponential excretion patterns in the declining segment (Fig. 7). With this one caution, one-compartment models are especially appropriate for estimating faecal output by pulse-dose methods (Ellis et al. 1980; Hunt et al. 1984; Krysl et al. 1988) because of their greater precision in estimating initial concentration $(C)$. In contrast to the integer gamma distributions used here, Roux \& Pienaar (1984) have proposed the use of non-integer-shape 
parameters in one-compartment models of digestion in and outflow from the reticulorumen. Although the non-integer forms have some advantages, the additional parameters which they involve pose additional problems in parameter estimation which are beyond the scope of the present paper.

\section{REFERENCES}

Austen, P., Buttle, H. L., Course, D. A., Cowie, A. T., Johnson, V. W., Oldham, J. D., Sutton, J. D. \& Watson, S. C. (1977). Zeitschrift fur Tierphysiologie, Tierenahrung und Futtermittelkunde 39, 192-197.

Balch, C. C. (1950). British Journal of Nutrition 4, 361-388.

Blaxter, K. L., Graham, N. Mc \& Wainman, F. W. (1956). British Journal of Nutrition 10, 69-71.

Brandt, C. S. \& Thacker, E. J. (1985). Journal of Animal Science 17, 218-223.

Broderick, G. A. (1978). Journal of Nutrition 108, 181-190.

Castle, E. J. (1956). British Journal of Nutrition 10, 15-23.

Deswysen, A. G. (1981). British Journal of Nutrition 46, 327-332.

Dhanoa, M. S., Siddons, R. C., France, J. \& Gale, D. L. (1985). British Journal of Nutrition 53, 663-671.

Ellis, W. C., Lascano, C. E. \& Matis, J. H. (1979). Annales de Recherche Veterinaire 10, 166-167.

Ellis, W. C., Matis, J. H., Pond, K. R., Lascano, C. E. \& Telford, J. P. (1984). In Herbivore Nutrition in the Subtropics and Tropics, pp. 269-293 [F. M. C. Gilchrist and R. I. Machie, editors]. Johannesburg: The Science Press.

Ellis, W. C., Matis, J. H., Rector, B. \& Rittenhouse, L. (1980). Journal of Animal Science 51, Suppl. 1, 235.

Faichney, G. J. (1984). In Control of Digestion and Metabolism in Ruminants, pp. 173-195. [L. P. Milligan, W. L. Grovum and A. Dobson, editors]. Englewood Cliffs: Prentice-Hall.

Faichney, G. J. \& Boston, R. C. (1983). Journal of Agricultural Science, Cambridge 101, 575-581.

Ferreiro, H. M., Boodoo, A. A., Sutton, J. D. \& Bishop, C. (1980). National Institute for Research in Dairying Report, p. 46. Shinfield, Reading: NIRD.

France, J., Thornley, J. H. M., Dhanoa, M. S. \& Siddons, R. C. (1985). Journal of Theoretical Biology 113, $743-758$

Grovum, W. L. \& Williams, V. J. (1973). British Journal of Nutrition 30, 313-329.

Hunt, C. W., Paterson, J. A., Miller, S. J. \& Williams, J. E. (1984). Journal of Animal Science 59, Suppl. 1, 427.

Jacquez, J. A. (1985). Compartmental Analysis in Biology and Medicine, 2nd ed. Ann Arbor: University of Michigan Press.

Krysi, L. J., Glayean, M. L., Estel, R. E. \& Sowell, B. F. (1988). Journal of Agricultural Science, Cambridge 111, $19-25$

Landaw, E. M. (1985). In Variability in Drug Therapy, pp. 187-200. [M. Rowland, L. B. Sheiner and J. L. Steimer, eds]. New York: Raven Press.

Luckey, T. C., Hartman, R., Know, T., Palmer, S., Kay, M.\& Terry, B. (1979). Nutrition Reports International 19, 561-571.

Luckey, T. C., Venugopal, B., Gray, D. \& Hutchinson, D. (1977). Nutrition Reports International 16, $339-347$.

Marcus, C. S. \& Lengemann, F. W. (1962). Journal of Nutrition 76, 179-182.

Matis, J. H. (1972). Biometrics 28, 597-602.

Matis, J. H. (1987). Journal of Theoretical Biology 124, 371-376.

Matis, J. H. \& Tolley, D. (1980). Federation Proceedings 39, 104-109.

Matis, J. H. \& Wehrly, T. E. (1979). Biometrics 35, 199-220.

Milne, J. A., MacRae, J. C., Spence, A. M. \& Wilson, S. (1978), British Journal of Nutrition 40, $347-357$.

Ørskov, E. R. \& McDonald, I. (1979). Journal of Agricultural Science, Cambridge 92, 499-503.

PCNONLIN (1985). PCNONLIN Users'Guide. Lexington, Ky: Statistical Consultants Inc.

Pond, K. R., Matis, J. H., Ellis, W. C. \& Satter, L. D. (1982). Federation Proceedings 41, 342.

Pond, K. R., Tolley, E. A., Ellis, W. C. \& Matis, J. H. (1984). In Techniques in Particle Size Analysis of Feed and Digesta in Ruminants, pp. 123-133. [P. M. Kennedy, editor]. Edmonton: Canadian Society of Animal Science.

Reid, C. S. W., John, A., Ulyatt, M. J., Waghorn, G. C.\& Milligan, L. P. (1979). Annales de Recherche Veterinaire 10, 205-207.

Roux, C. Z. \& Pienaar, J. P. (1984). In Techniques in Particle Size Analysis of Feed and Digesta in Ruminants, p. 176 [P. M. Kennedy, editor]. Edmonton: Canadian Society of Animal Science.

SAS (1979). SAS Users' Guide. Cary, North Carolina: Statistical Analysis Systems Institute Inc.

Sutherland, J. M. (1988). In Aspects of Digestive Physiology in Ruminants, pp. 43-73 [A. Dobson and M. H. Dobson, editors]. Ithaca, N.Y.: Cornell University Press.

Waldo, D. R., Smith, L. W. \& Cox, E. L. (1972). Journal of Dairy Science 55, 125-129.

Warner, A. C. I. (1981). Nutrition Abstracts and Reviews. Ser. B 51, 789-820. 